\title{
Optical and infrared observations of the young SMC blob N26 and its environment ${ }^{\star}, \star \star$
}

\author{
G. Testor ${ }^{1, \star \star \star}$, M. Heydari-Malayeri ${ }^{1}$, C.-H. R. Chen ${ }^{2,3}$, J. L. Lemaire ${ }^{1,4, \star \star \star}$, M. Sewiło ${ }^{5,6}$, and S. Diana ${ }^{4}$ \\ 1 LERMA, UMR 8112 du CNRS, Observatoire de Paris, 92195 Meudon, France \\ e-mail: gerard.testor@obspm.fr \\ 2 Department of Astronomy, University of Virginia, PO Box 400325, Charlottesville VA 22904, USA \\ 3 Max-Planck-Institut fur Radioastronomie, 53121 Bonn, Germany \\ ${ }^{4}$ Université de Cergy-Pontoise, 95031 Cergy Cedex, France \\ 5 The Johns Hopkins University, Department of Physics and Astronomy, 366 Bloomberg Center, 3400 N. Charles Street, \\ Baltimore MD 21218, USA \\ ${ }^{6}$ Space Science Institute, 4750 Walnut St. Suite 205, Boulder CO 80301, USA
}

Received 18 November 2011 / Accepted 6 February 2014

\section{ABSTRACT}

\begin{abstract}
Context. High-excitation compact $\mathrm{H}$ II regions of the Magellanic Clouds are sites of recent massive star formation in low metallicity environments.

Aims. Detailed study of these regions and their environments using high-spatial resolution observations is necessary to better understand massive star formation, which is still an unsolved problem. We aim at a detailed study of the Small Magellanic Cloud compact H II region N26, which is only $\sim 4^{\prime \prime}$ in diameter.

Methods. This study is based on high spatial resolution imaging ( 0'.1-0'3) in $J H K$ s and $L^{\prime}$ bands, using the VLT equipped with the NAOS adaptive optics system. A larger region $(\sim 50 \mathrm{pc} \times 76 \mathrm{pc})$ was also imaged at medium spatial resolution, using the ESO $2.2 \mathrm{~m}$ telescope in optical wavelengths. We also used the $J H K$ s archival data from the IRSF survey and the Spitzer Space Telescope SAGESMC survey.

Results. Our high-resolution $J H K$ s data of the compact high-excitation $\mathrm{H}_{\text {II }}$ region N26 reveal a new, bright component (C) between the two already known optical components $\mathrm{A}$ and $\mathrm{B}$. Components $\mathrm{A}$ and $\mathrm{C}$ are resolved into several stars. Component $\mathrm{A}$ is the main ionization source of $\mathrm{N} 26$ and coincides with the radio continuum source B0046-7333. A new compact $\mathrm{H}_{\text {II }}$ region with very faint [O $\mathrm{III}] \lambda 5007$ emission has been discovered. In the mid-infrared, our field resembles a shell formed by filaments and dust clumps, coinciding with the molecular cloud SMCB2. Region N22, located in the center of the shell, is the most excited $\mathrm{H}_{\text {II }}$ region of the complex and seems to have created a cavity in SMCB2. We derive nebular parameters from spectra, and using color-magnitude and color-color diagrams, we identify stellar sources that show significant near-infrared excess emission in order to identify the best YSO candidates.
\end{abstract}

Key words. Magellanic Clouds - ISM: individual objects: N26 - HII regions - dust, extinction - stars: formation - stars: massive

\section{Introduction}

Although they are rare and short-lived, massive stars $\left(>8 M_{\odot}\right)$ play an important role in several fields of astrophysics. However, their formation process is still an unsolved problem in spite of progress, both in theory and observation, in recent years. The Magellanic Clouds (MCs) offer valuable opportunities for this study since they are seen nearly face-on and have welldetermined distances (Keller \& Wood 2006; Schaefer 2008). This facilitates obtaining accurate absolute magnitudes and fluxes. Moreover, their overall extinction is low (Massey et al. 1995; Westerlund 1997; Bonanos et al. 2010). Being metal-poor (Peña-Guerrero et al. 2012), they provide important templates for studying star formation in distant metal-poor galaxies which cannot be observed with comparable spatial resolution.

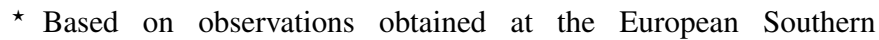
Observatory, El Paranal, Chile.

$\star \star$ Full Table 2 is only available at the CDS via anonymous ftp to cdsarc.u-strasbg.fr (130.79.128.5) or via

http://cdsarc.u-strasbg.fr/viz-bin/qcat?J/A+A/564/A31

$\star \star \star$ Visiting astronomer at VLT Paranal.
The youngest massive stars in the MCs accessible to infrared and optical observations are found in high-excitation blobs (HEBs, Heydari-Malayeri \& Testor 1982, 2010, and references therein). This is a rare class of $\mathrm{H}_{\text {II }}$ regions in the MCs; so far only six members have been detected in the Large Magellanic Cloud (LMC) and five in the Small Magellanic Cloud (SMC). For massive stars the accretion time-scale is larger than the Kelvin-Helmholtz time-scale. This means that massive stars reach the main sequence while accretion is still going on. Moreover, they evolve very fast. Therefore, obtaining the physical parameters of massive stars at their birth may be an unattainable task. Consequently, HEBs offer a compromise between stars inside ultra-compact $\mathrm{H}$ II regions and the exciting stars of evolved $\mathrm{H}_{\text {II }}$ regions.

In contrast to the typical $\mathrm{H}_{\text {II }}$ regions of the MCs, which are extended structures (sizes of several arc minutes corresponding to more than $50 \mathrm{pc}$, powered by a large number of exciting stars), HEBs are very dense, small regions $\left(\sim 4^{\prime \prime}\right.$, to $10^{\prime \prime}$, in diameter corresponding to $\sim 1-3 \mathrm{pc}$ ). They have a higher degree of excitation $([\mathrm{O} \mathrm{III}] / \mathrm{H} \beta])$ with respect to the typical $\mathrm{H}$ II regions, and are, in general, heavily affected by local dust. In comparison with Galactic regions, some of the HEBs are similar 

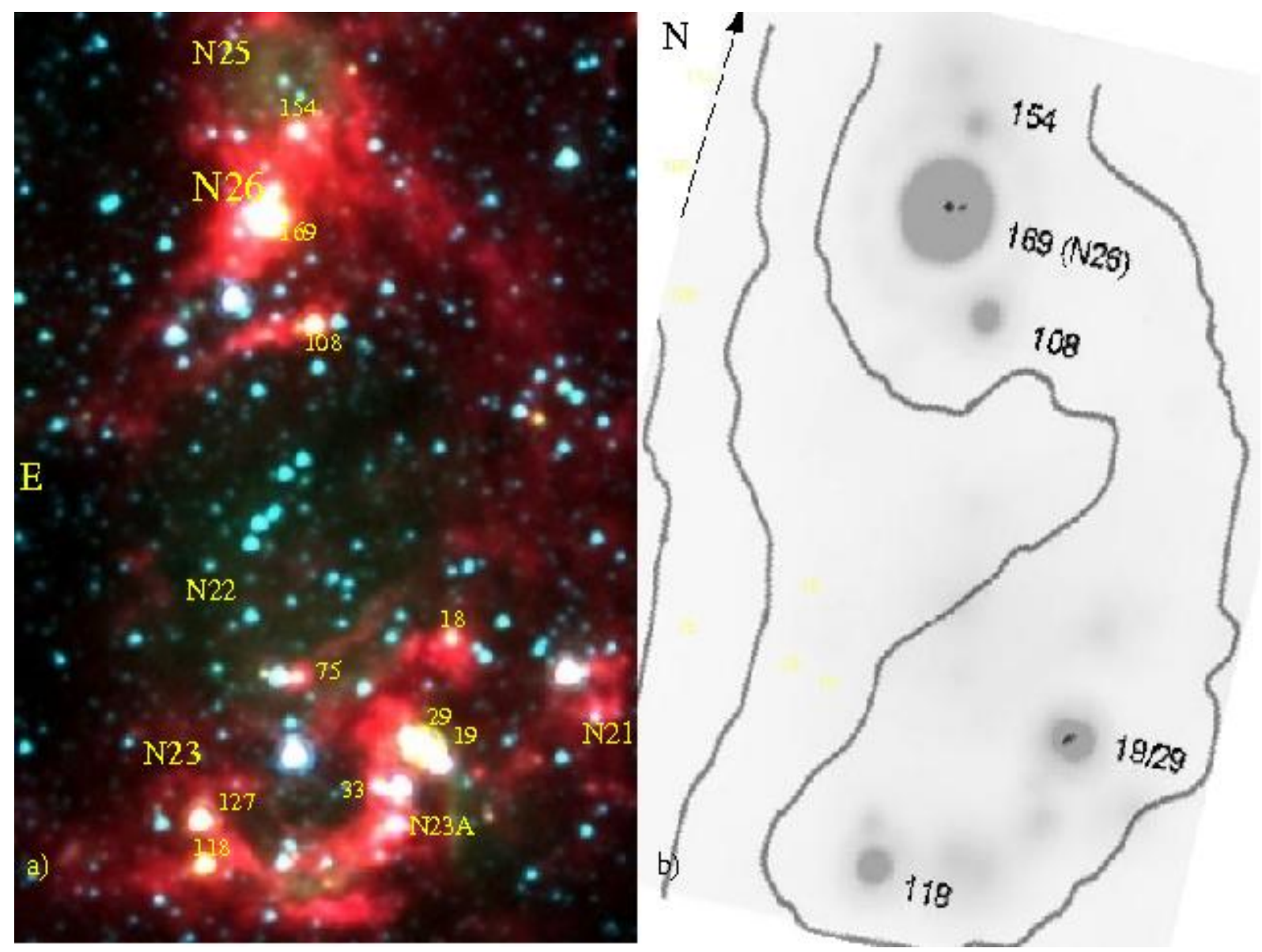

Fig. 1. a) False-color composite image of SMCB2-H II from Spitzer IRAC bands $3.6 \mu \mathrm{m}$ (blue), $4.5 \mu \mathrm{m}$ (green), and $8 \mu \mathrm{m}$ (red). The various H II regions detected by Henize (1956) in this part of the SMC are labeled, as well as the YSO candidates (Sect. 4.6). The image center is $\alpha=0^{\mathrm{h}} 48^{\mathrm{m}} 01^{\mathrm{s}} .85, \delta=-73^{\circ} 16^{\prime} 03^{\prime \prime} .75$. Total field size $168^{\prime \prime} \times 251^{\prime \prime}$ or $50 \times 76 \mathrm{pc}$. b) The MIPS $24 \mu \mathrm{m}$ image of the SMCB2-HII region with the CO contours overlaid. The contours outline the molecular cloud SMCB2 (Bot et al. 2010). The bright stars are labeled (see electronic edition of the journal for a color version of this figure).

to classical $\mathrm{H}$ II regions and some look like compact $\mathrm{H}$ II regions (Martin-Hernandez 2005). However, HEBs should be considered in the context of massive star formation in the MCs. Compared with other compact $\mathrm{H}_{\text {II }}$ regions of the same sizes in the MCs, they constitute a distinctly detached group with high excitations and luminosities (Meynadier et al. 2007).

This paper focuses mainly on N26 (LHA 115-N26, Henize 1956), a HEB situated in the SMC, the first study of which was presented by Testor (2001). Its (J2000) coordinates are $\alpha=0^{\mathrm{h}} 48^{\mathrm{m}} 08^{\mathrm{s}} \cdot 60, \delta=-73^{\circ} 14^{\prime} 54^{\prime \prime} .^{\prime} 69$. It is a compact $\mathrm{H}_{\mathrm{II}}$ region of $\sim 4 "$, or $1 \mathrm{pc}$, in diameter composed of two components, $\mathrm{A}$ and $\mathrm{B}$. This object is highly excited, with a ratio of [O III] $\lambda \lambda 4959+5007 / \mathrm{H} \beta$ reaching 8 in component A (Fig. 6 in Testor 2001). Region N26 has an ultra-compact H II region counterpart detected with high-resolution $6 \mathrm{~cm}$ radio continuum observations (Indebetouw et al. 2004). From optical spectra, the exciting source of component A was classified as 07-9 V (Testor 2001). The extinction in N26 is relatively high, reaching an $E(B-V)$ of $\sim 1 \mathrm{mag}$ in the direction of component B. That first study used relatively low-resolution observations and only in the optical range.

Region N26 is located in the southwest part of the bar of the SMC, where several $\mathrm{H}_{\text {II }}$ regions are visible in its close vicinity. Figure 1a shows N25 to the north and N22, N21, and N23 to the south of N26. The largest and the most diffuse H II region of the group is $\mathrm{N} 22$ around which the other $\mathrm{H}$ II regions are distributed. These H II regions are associated with embedded clusters (Bica \& Dutra 2000), and are also detected in the $\mathrm{H} \alpha$ survey conducted by Davies et al. (1976). Region N26 is the most compact and smallest $\mathrm{H}_{\mathrm{II}}$ region in the group, which suggests that it is the youngest among them. A giant molecular cloud labeled SMCB2 (Fig. 1b) is detected toward this area of the SMC (Rubio et al. 1993). The molecular cloud presents three ${ }^{12} \mathrm{CO}(1-0)$ peaks, one to the north and two to the south, the velocities of which range from $108 \mathrm{~km} \mathrm{~s}^{-1}$ to $126 \mathrm{~km} \mathrm{~s}^{-1}$; this suggests that they are likely to be physically associated. Bot et al. (2010) observed SMCB2 with the LABOCA camera on the APEX telescope at $870 \mu \mathrm{m}$ and found a dust temperature of $12 \mathrm{~K}$, a mass of $M_{\mathrm{H}}^{870 \mu \mathrm{m}}=8.5 \times 10^{5} M_{\odot}$ for the northern peak SMCB2-N, and $65 \times 10^{4} M_{\odot}$ for the southern peak SMCB2-S (Fig. 1). Far infrared and millimeter observations of the region have been performed by Wilke et al. (2003) and Mizuno et al. (2001), respectively. The Spitzer data base has also been used to study point sources in the IRAC and MIPS data (Bolatto et al. 2007; Lawton et al. 2010) of our region as part of the Spitzer Survey of the Small Magellanic Cloud (S3MC).

Star forming regions like the N26 area are expected to be associated with young stellar objects (YSOs). The use of $J H K$ color-magnitude and color-color diagrams are useful tools for detecting the candidates. A more suitable wavelength for determining the nature of the IR-excess is the $L$-band, which increases the IR-excess and reduces the contribution of extended emission 

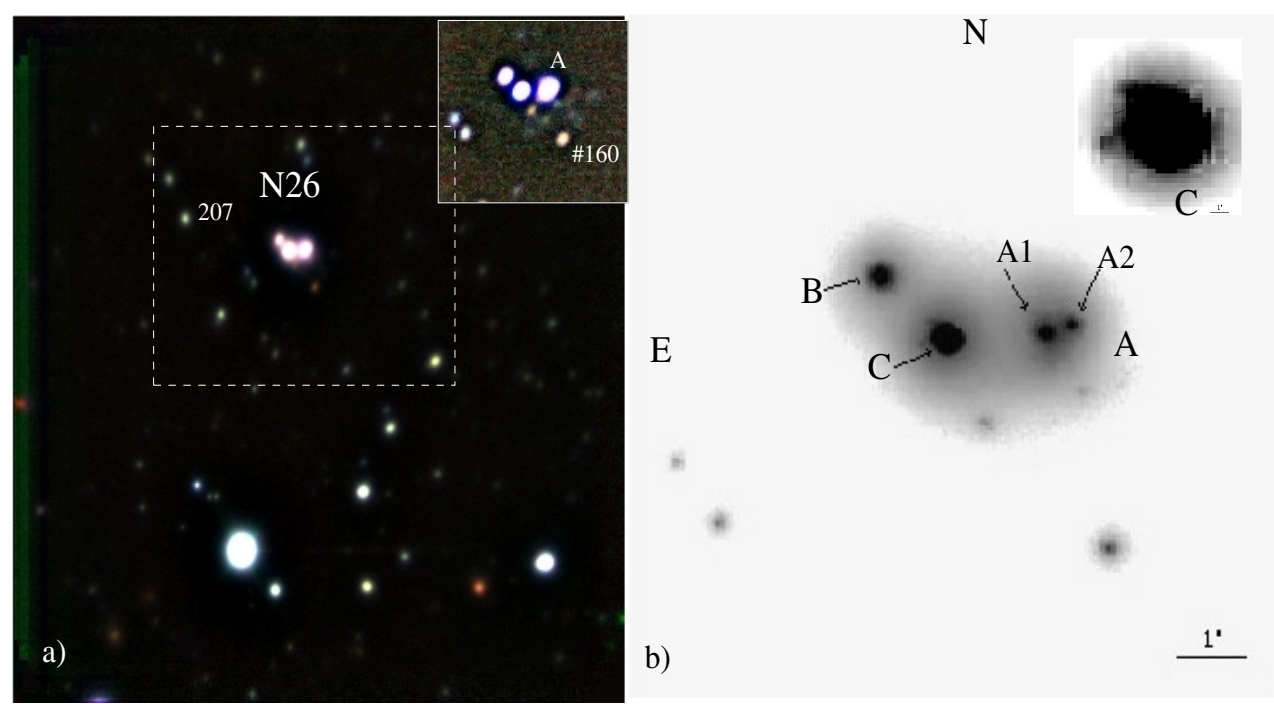

Fig. 2. a) False-color image of the $\mathrm{N} 26 \mathrm{H}$ in region composed of $J$ (blue), $H$ (green), and $K$ (red) bands obtained with the S54 camera. The very faint cluster enhanced by the DDP technique seen in the inset (top right) is located between component A and star 160 (see Fig. 3). The field size is $58^{\prime \prime} \times 53^{\prime \prime}$ or $18 \times 16 \mathrm{pc}$ and the small area is shown in dashed lines. Star 207 is the IRSF source 0481111-73145210 used in the photometry calibration (Sect. 2.1.1). b) Image of N26 in the Ks band, obtained with the S27 camera, also processed with the same technique. The field size is $10^{\prime \prime} \times 9$ '. 5 or $3.0 \mathrm{pc} \times 2.7 \mathrm{pc}$. The inset of size 1." $6 \times 1$ 1.' 6 shows an enlarged view of component C. North is up and east left (see electronic edition of the journal for a color version of this figure).

from reflection nebulae and HiI regions (Lada et al. 2000). However, near- and mid-IR color-magnitude and color-color diagrams obtained from IRAC data are still better tools for identifying the origin of the reddening of embedded stars (Allen et al. 2004; Bolatto et al. 2007). In this paper we present the results for the whole area using $B V J H K$ s, 3.6, 4.5, 5.8, 8.0, 24, 70, and $160 \mu \mathrm{m}$ bands in mid-spatial resolution photometry, as well as the $J H K$ - and $L$ '-band high spatial resolution observations from adaptive optics.

The paper is arranged as follows. In Sect. 2, we present the instruments employed for these observations, the reduction procedures used as well as the archival data. In Sect. 3 we describe the results and analysis of the observations. In Sect. 4, we discuss the results. Concluding remarks are given in Sect. 5 .

\section{Observations and data reduction}

\subsection{Imaging and photometry}

\subsubsection{VLT/NACO}

Near-IR observations of a small area centered on the SMC compact $\mathrm{H}_{\text {II }}$ region N26 were obtained at the ESO VLT on October 9 and 10, 2004. Images and spectra were taken using NACO on UT4, composed of the Nasmyth Adaptive Optics System (NAOS) and the High Resolution IR Camera and Spectrometer (CONICA). The detector was a $1026 \times 1024$ SBRC InSb Aladdin 3 array. The cameras S54 (Fig. 2a) and S27 (Fig. 2b) were used in the range $1.0-2.5 \mu \mathrm{m}$ and the L27 camera in the range $2.5-5.0 \mu \mathrm{m}$.

The field of view (FOV) of the S54 camera was 54" $\times 54^{\prime \prime}$ with a pixel size of 0 .' $^{\prime} 05274$, corresponding to $0.015 \mathrm{pc}$ at the distance modulus of $m-M=18.93$ mag for the SMC (Keller \& Wood 2006). The FOV of the S27 and L27 camera was 27 "' $15 \times$ 27 .' 15 with a pixel size of $0 .{ }^{\prime} 02637$, corresponding to $0.0075 \mathrm{pc}$. As adaptive optics (AO) reference source for wavefront sensing we used the 2MASS star (J200004809.84-731518.5) of magnitude $K=10.370$ (Cutri et al. 2003). The conditions were photometric, and the seeing ranged from 0.65 to $1^{\prime \prime}$ in the visible.
Table 1. Log of VLT near-IR CCD images of SMC N26.

\begin{tabular}{|c|c|c|c|c|}
\hline Filter & $\begin{array}{c}\text { Exposure } \\
t(\mathrm{~s}) \times n\end{array}$ & Mode & $\begin{array}{c}\text { Seeing } \\
\left({ }^{\prime \prime}\right)\end{array}$ & $\begin{array}{c}F W H M \\
\left({ }^{\prime \prime}\right)\end{array}$ \\
\hline$J 1.27 \mu \mathrm{m}$ & $20 \times 30$ & S54 & $0.6-0.9$ & 0.35 \\
\hline$H 1.66 \mu \mathrm{m}$ & $4 \times 30$ & & & 0.27 \\
\hline$K \mathrm{~s} 2.18 \mu \mathrm{m}$ & $5 \times 30$ & "I & "I & 0.21 \\
\hline$K \mathrm{~s} 2.18 \mu \mathrm{m}$ & $60 \times 1$ & S27 & $0.9-1.2$ & 0.10 \\
\hline$L^{\prime} 3.8 \mu \mathrm{m}$ & $0.18 \times 30$ & & $0.9-1.2$ & 0.10 \\
\hline
\end{tabular}

Broadband $J H K$ s images were obtained with the S54 camera. Images with higher spatial resolution in the $K$ s and $L^{\prime}$ bands were also obtained with the S27 camera. The $L^{\prime}$ photometry was extracted only for the three brightest components of N26 (Fig. 4). The $L^{\prime}$ magnitudes for all other stars are the $3.6 \mu \mathrm{m}$ data taken from the IRAC catalog (see Sect. 2.3). The log of the near-IR imaging observations is given in Table 1. The AutoJitter mode was used; that is, the telescope moves at each exposure according to a random pattern in a $6^{\prime \prime} \times 6^{\prime \prime}$ box. Table 1 lists the stellar full width at half maximum (FWHM) in final images of different observed bands. The adaptive optics image is affected by anisoplanatism when the reference star is not on axis and leads to a degradation of the point spread function (PSF) becoming more elongated as the angular offset from the reference star increases. The data were reduced mainly with the ESO software packages MIDAS and ECLIPSE. A false-color image of N26 combining the $J$ (blue), $H$ (green), and $K$ s (red) bands is shown in Fig. 2.

The high resolution of NACO allowed us to separate the crowded components in N26. However, its distance from the the AO reference star, $\sim 30^{\prime \prime}$, increases the anisoplanatism effects and so photometry will be performed on a small area $\left(30^{\prime \prime} \times 25^{\prime \prime}\right)$ around N26. In that area the instrumental magnitudes of the stars were derived, using the DAOPHOT multiple-simultaneousprofile-fitting photometry routine (NSTAR; Stetson 1987), which is well adapted for photometry in crowded fields. The uncertainties of the instrumental magnitudes $\left(m_{\mathrm{e}}\right)$ in the $J, H$, and $K$ s bands are less than 0.03 mag for stars with $K \mathrm{~s}<16.5 \mathrm{mag}$, 


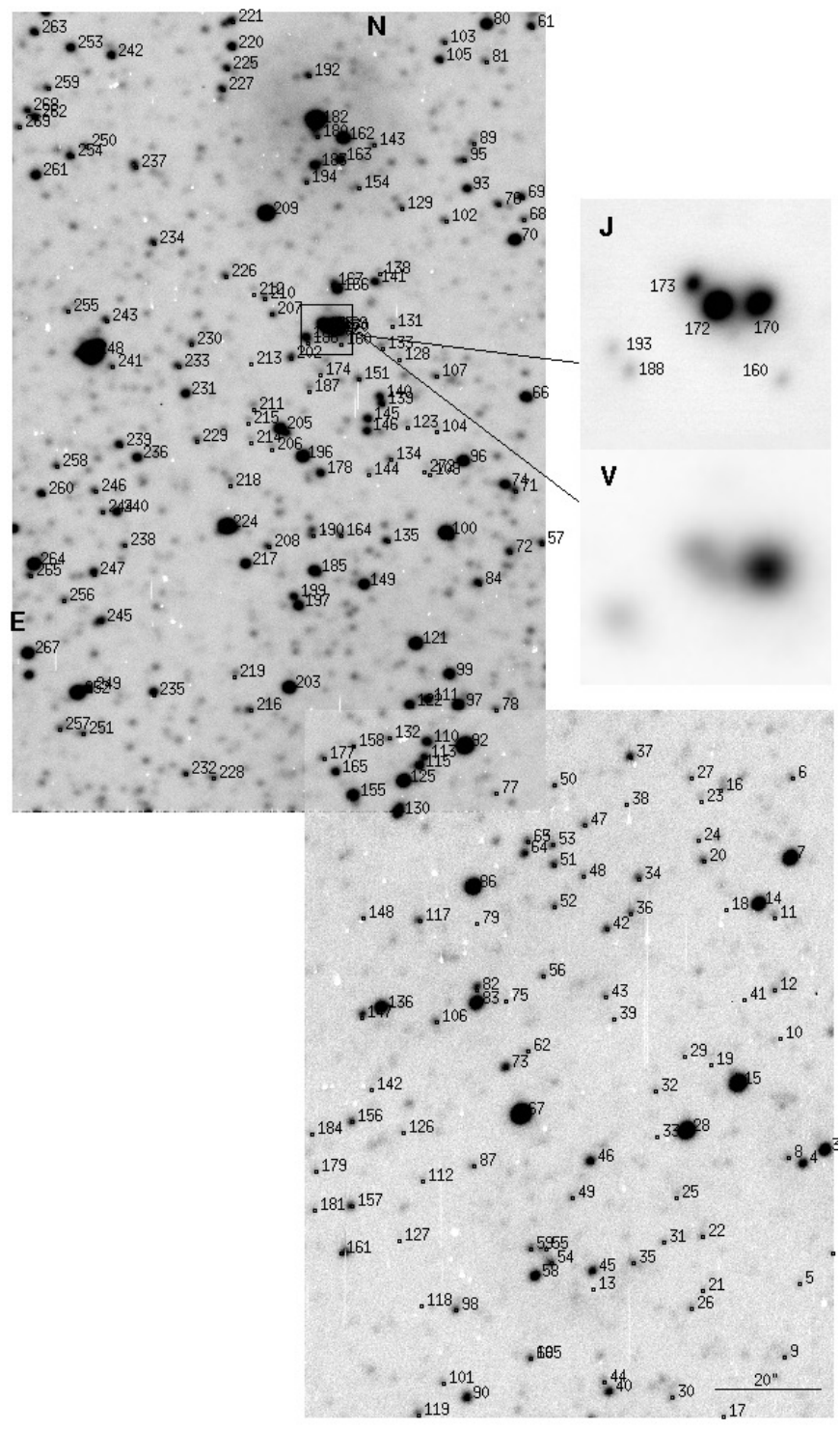

Fig. 3. An optical image in the $V$ band obtained with the ESO $2.2 \mathrm{~m}$ telescope. The numbers refer to Table 2. The field size is $153^{\prime \prime} \times 264^{\prime \prime}$ or $\sim 46 \mathrm{pc} \times 80 \mathrm{pc}$. A section of the field containing the $\mathrm{H}$ iI region N26 is outlined. It is 9.' $6 \times 9$.' 6 and shows a blow-up of the $J$ and $V$ images obtained with the NACO (S54 camera) and the $2.2 \mathrm{~m}$ telescope, respectively.

less than 0.06 for stars with $16.5<K \mathrm{~s}<18 \mathrm{mag}$, and greater than 0.1 for stars $18<K s<20$ mag. In this small area, the anisoplanatism effects become negligible, but because of the $m_{\mathrm{e}}$ of about $0.04 \mathrm{mag}$ on the faint isolated stars used as PSF, the accuracy of the photometry is slightly degraded.

In the small area centered on N26 (Fig. 2, inset), we show for the first time the presence of six stars embedded in the H II region N26. These stars, shown in Fig. 3 (inset), do not have IRSF counterparts. Two IRSF stars (Kato et al. 2007) were available: 0481111-73145210, corresponding to our star 207, and IRSF 0481032-731459, corresponding to our star 202. As their colors are the same, we could not calculate color correction, which adds uncertainty to the YSO SED calculation. The resulting photometry for these six stars in all bands has errors of 0.02 to 0.04 for $K \mathrm{~s}<15.5 \mathrm{mag}$ and 0.04 to 0.09 for $K \mathrm{~s}>15.5 \mathrm{mag}$. These are square root errors derived from IRSF accuracies available only for star 207 (Kato et al. 2007) and our instrumental uncertainties. More specifically, we applied the IRSF inaccuracy of star 207 to the six stars in the sample. This means that since 207 is fainter than stars 170, 172, and 173, we overestimate the final uncertainties on these stars. The other JHKs magnitudes of the whole field are extracted from the IRSF catalog (see Sect. 2.3).

Table 2 presents the data set that includes our $J H K \mathrm{~s} L$ ' NACO photometry (Sect. 2.1.1), $B V$ data (Sect. 2.1.2), as well as the archival data from IRSF (JHKs) and SAGE-SMC (3.6-160 $\mu \mathrm{m}$, Sect. 2.3). This table contains only sources brighter than $J=$ 18.8 both for IRSF and NACO photometry, corresponding to a signal to noise of $>10$. Column 1 gives the identification numbers, which are our serial numbers. The various identification numbers in the archival data for each star can be obtained from the corresponding coordinates. The main stars dealt with in this study (Table 2) do not have other identification numbers. Columns 3 and 4 list the corresponding J2000.0 coordinates. Columns 5 to 28 give the magnitudes $B, V, J, H, K s, 3.6,4.5,5.8$, $8.0,24,70$, and $160 \mu \mathrm{m}$ with their uncertainties.

\subsection{2. $2.2 \mathrm{~m}$ telescope}

We used $B$ and $V$ broad bands as well as narrow emission line bands $\left(\mathrm{H} \beta,\left[\mathrm{O}_{\mathrm{III}}\right] \lambda_{5007)}\right.$ to observe the regions N21, N22, N23, $\mathrm{N} 25$, and N26. The observations were made in October 1987 using the $2.2 \mathrm{~m}$ telescope at La Silla. The detector was a $1000 \times$ 1000 CCD (GEC) with a pixel size of 0.26 on the sky. The seeing was $\sim 1$ ". 5 and exposure times ranged from $90 \mathrm{~s}$ to $900 \mathrm{~s}$.

The $B V$ data reduction of the two overlapping frames containing our field (Fig. 3) was done using DAOPHOT/ALLSTAR. The photometric calibration was carried out using eight bright and isolated stars extracted from Massey's (2002) catalog. They belonged to two samples situated toward the regions N22 in the south and N26 in the north. The $V$ magnitudes ranged from 13.06 to 15.03 and the corresponding $B-V$ colors had a value between $-0.06 \mathrm{mag}$ and $1.59 \mathrm{mag}$. The zero point and color correction relationships, derived from the linear regression method, were $V=v+0.05(B-V)-0.03$ (s.d. \pm 0.04 ) and $B-V=0.98$ $(b-v)-0.02$ (s.d. \pm 0.03 ). The total square root errors on $B$ and $V$ (Table 2) were obtained using our instrumental errors and the corresponding average statistical errors given in Table 2 of Massey (2002).

\subsection{Spectroscopy}

\subsubsection{NACO spectroscopy}

Spectroscopy of N26 was performed (October 9, 2004) in the S54-4-SHK mode. The spectral range was $1.30 \mu \mathrm{m}$ to $2.60 \mu \mathrm{m}$, the linear dispersion $1.94 \mathrm{~nm} / \mathrm{pixel}$, and spatial scale 53 mas/pixel. The seeing varied between $0 .{ }^{\prime} 6$ and $0{ }^{\prime} 9$. One EW long-slit spectrum S1 crossing component A of N26 was taken with NACO. The slit width was 172 mas and the spectral resolution $\sim 400$. For each exposure the detector integration time (DIT) was $100 \mathrm{~s}$. Ten exposures were obtained in the AutoNod on Slit mode, which allows the spectroscopy of moderately extended objects. In order to remove telluric absorption features, stars with a similar airmass were observed as telluric standards. Spectroscopy was reduced with the MIDAS software package LONG. 

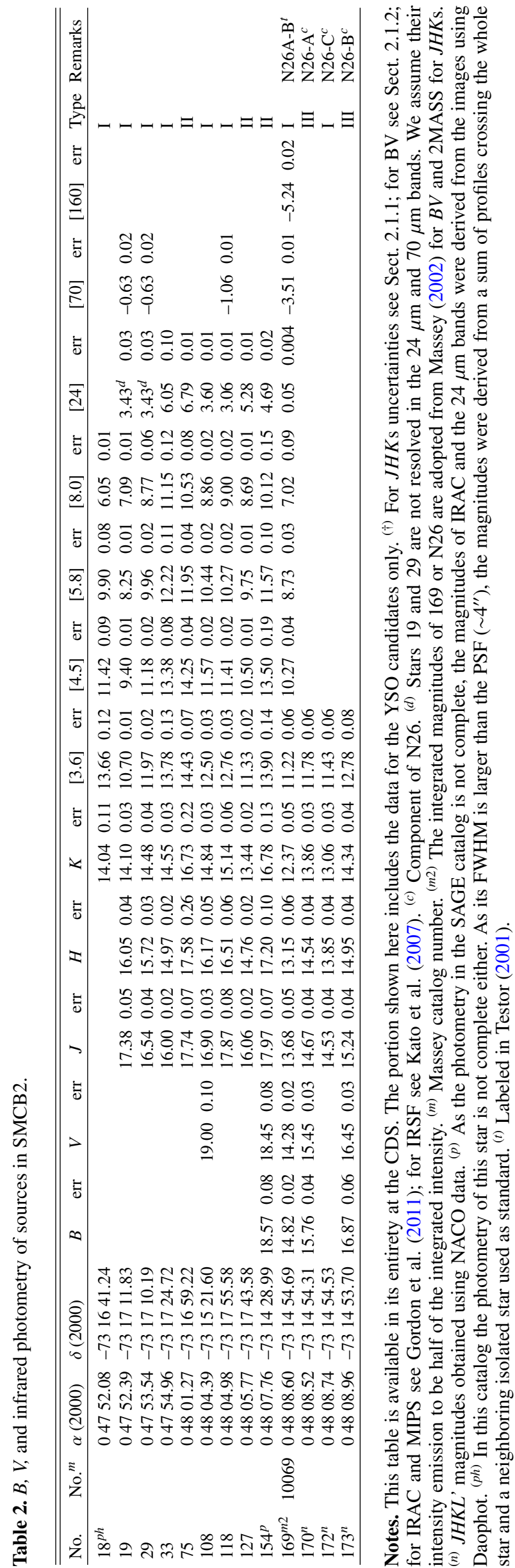

\subsubsection{Boller and Chivens spectroscopy}

The optical spectroscopic observations were performed at the ESO $1.5 \mathrm{~m}$ telescope (September 1987) with the Boller \& Chivens spectrograph (Heydari-Malayeri et al. 1989). Grating 25 covered from $4300 \AA$ to $6800 \AA$ with a dispersion of $170 \AA / \mathrm{mm}$ or 1 .' $27 /$ pix (binned $\times 2$ ). The spectra were obtained through a $4^{\prime \prime}$ slit width oriented east-west, in the directions $a, b, c, d$, and $e$ (Fig. 7) (see Sect. 4.4.1). Exposure times were 1800 s. The flux was calibrated using observations of two spectrophotometric standard stars, LTT7987 and L870-2.

\subsection{Archival data and matching}

The Spitzer observations of the working field were obtained as part of the Legacy Program Surveying the Agents of Galaxy Evolution in the Tidally-Disrupted, Low-Metallicity Small Magellanic Cloud (SAGE-SMC; Gordon et al. 2011). These observations include images taken with the IRAC 3.6, 4.5, 5.8, and $8.0 \mu \mathrm{m}$ bands and MIPS 24, 70, and $160 \mu \mathrm{m}$ bands. The $L$-band photometry used in this work refers to the $3.6 \mu \mathrm{m}$ band. The details of observations and data processing are given in Gordon et al. (2011). The IRAC and MIPS $24 \mu \mathrm{m}$ photometry of sources in the working field are adopted from the SAGE-SMC point source catalog. The IRAC resolution ranged from $1^{\prime \prime}$. 7 to $2^{\prime \prime}$ $(0.5 \mathrm{pc}$ to $0.6 \mathrm{pc})$, those for MIPS images from $6^{\prime \prime}$ to $40^{\prime \prime}$ (1.8 pc to $12 \mathrm{pc})$.

We also used the data obtained with the InfraRed Survey Facility (IRSF) (Kato et al. 2007). The IRSF has a pixel scale of 0.45 , average seeing of $1^{\prime \prime} .3,1^{\prime \prime} .2$, and $1^{\prime \prime} .1$ in the $J H K$ s bands respectively, and limiting magnitudes of $J=18.5, H=18.2$, and $K \mathrm{~s}=17.4 \mathrm{mag}$ for the SMC. For star $169, B V$ and $J H K$ data were extracted from the catalogs of Massey and 2MASS respectively. Table 2 presents the data set that includes our $B V$ (see Sect. 2.1.2) and $J H K s L^{\prime}$ NACO photometry and archival data from IRSF (JHKs) and SAGE-SMC (3.6-160 $\mu \mathrm{m})$.

The matching of the new data with those of older data from different catalogs was not carried out automatically. Because of the crowding as well as low magnitudes in some wavelengths, the star labels (identification numbers) were matched manually. To do this, we used the Aladin tool ${ }^{1}$ to visualize our images in different wavelengths and superimpose the labels of the stars. Thus, for each star we obtained a table line including several labels corresponding to the star in different wavelengths.

The transformation from the chip $x, y$ positions to the equatorial right ascension (RA) and declination (Dec) coordinates, listed in Table 2, was carried out using three stars in each $V$ frame. To calibrate the coordinates, we used Massey's catalog (2002), which has an astrometric accuracy of $\sim 0$. $^{\prime} 4$. Our final coordinates are accurate within $\sim 0$ '. 5 . For stars with no counterpart in the $V$ frame, as identified by eye, we adopted the coordinates given by Kato et al. (2007) and the IRAC catalog, which have an accuracy of $0 !^{\prime} 1$ and 0.25 , respectively. In the particular case of stars 170,172 , and 173, the transformation was obtained using neighboring stars of the Kato catalog, leading to an accuracy of $\sim 0 . ' 2$.

\section{Results}

\subsection{General morphology of N26}

The high spatial resolution provided by NACO reveals N26 as a compact $\mathrm{H}_{\mathrm{II}}$ region of radius $\sim 2^{\prime \prime}$, which is one of the most

1 http://aladin.u-strasbg.fr 


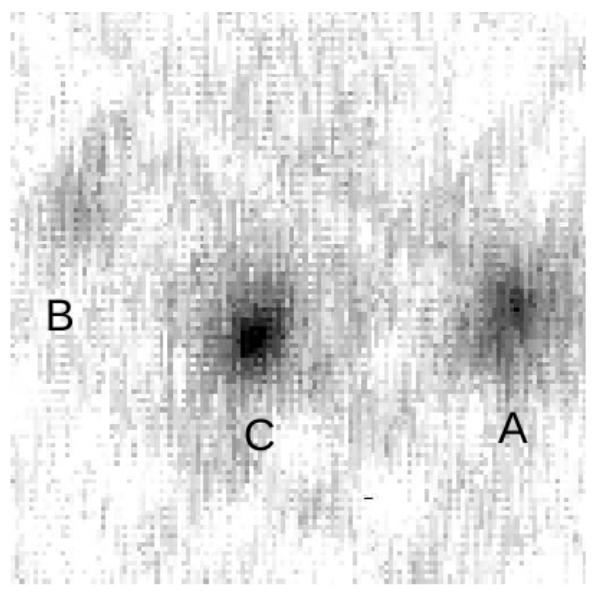

Fig. 4. An underexposed image of $\mathrm{N} 26$ in the $L^{\prime}$ band (3.6 $\left.\mu \mathrm{m}\right)$ showing the three components A, C, and B. They correspond to stars 170, 172, and 173, respectively. The field size is 3 '. $4 \times 3$ 3' 4 ; north is up and east right.

compact HEBs so far detected. It also confirms the presence of two bright nebular components, A and B, separated by 2.'7, as first detected by Testor (2001) in optical wavelengths.

Moreover, the NACO observations uncover a well-resolved red component located between A (170) and B (173), which we call C (172) (Fig. 4). Up to now, no optical counterpart has been detected for component $C$. Using a digital development process $(\mathrm{DDP})^{2}$, a very faint star cluster of diameter $\sim 4^{\prime \prime}$ (Fig. 2a) appears between component $A$ and the faint red star 160 . On the Ks image (Fig. 2b) this cluster is not detected and component A seems to be formed mainly by two bright stars, A1 and A2, separated by 0.39 . The new component $C$, at $1 . \prime 48$ to the east of A1, seems to be surrounded by at least two faint stars, as shown in the inset of Fig. $2 b$.

The east-west NACO spectrum S1 (Fig. 5) crossing components $\mathrm{A}$ and $\mathrm{C}$ gives the distribution of the intensity in the $\operatorname{Br} \gamma 2.17 \mu \mathrm{m}$, He $\mathrm{I} 2.05 \mu \mathrm{m}$, and $\mathrm{H}_{2}$ lines along the slit. The large profile of $\mathrm{A}$ allows the assumption that it contains more than two stars. The $\mathrm{H}_{2}$ emission is mainly centered on the red component $\mathrm{C}$ and spans over 3".5. Figure 6 shows the intensity profiles of the emission lines $\operatorname{Br} \gamma 2.17 \mu \mathrm{m}, \mathrm{He}_{\mathrm{I}} \lambda 2.05 \mu \mathrm{m}$, and $\mathrm{H}_{2} \lambda 2.12 \mu \mathrm{m}$ for each component. These 1D spectra are extracted by summing 8 and 4 pixels centered on the maximum intensity of $\mathrm{A}$ and $\mathrm{C}$ along the slit of $\mathrm{S} 1$. The corresponding relative intensities are listed in Table 3.

\section{2. lonizing source of N26}

From the above-mentioned spectrum S1 a $\mathrm{He}$ I $\lambda 2.11 / \mathrm{Br} \gamma \lambda 2.17$ ratio of $\sim 0.033$ is derived for component A (Table 3). According to Hanson et al. (2002), this ratio corresponds to an ionizing source of type between $\mathrm{O} 7 \mathrm{~V}$ and late $\mathrm{O}$ stars. This is in agreement with the result obtained by Testor (2001), who, on the basis of optical spectroscopy, derived a spectral type 07-O9 V, from the integrated light for components A, B, and the newly detected component $\mathrm{C}$. We note that this integrated entity will be labeled 169 on the color-magnitude and color-color diagrams presented in the following section. It appears that the main ionizing source should be embedded in component $\mathrm{A}$ and that the ionization of $B$ and $C$ is negligible. Another piece of support for this deduction comes from high-resolution radio continuum

2 http://www .asahi-net.or.jp/ rt6k-okn/its98/ddp1.htm

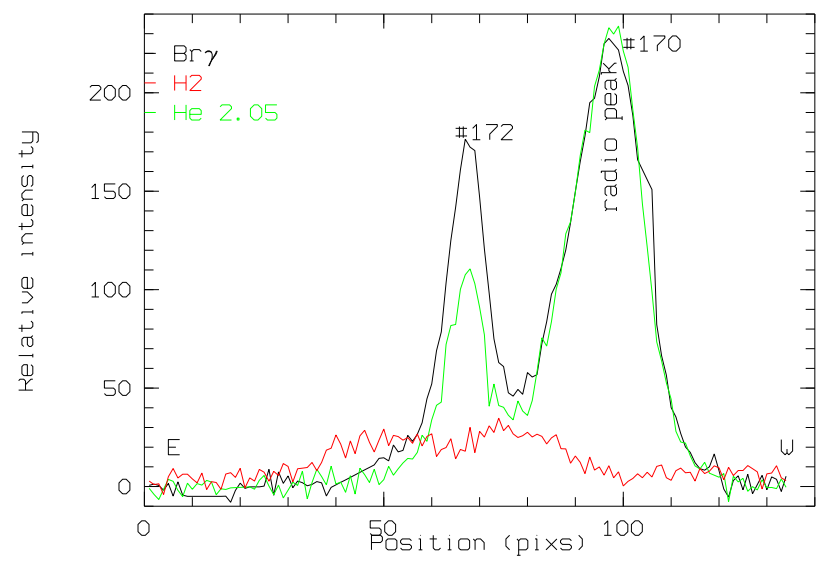

Fig. 5. The intensity profiles of near-IR emission lines from a NACO long-slit spectrum in east-west direction (left-right) crossing stars 170 (component A) and 172 (component C). Star 173 (component B) does not appear because it is not situated on the E-W cross-cut. The red line represents the $\mathrm{H}_{2} 2.121 \mu \mathrm{m}$ emission line. The green line represents the He I $2.113 \mu \mathrm{m}$ and the black line the $\mathrm{Br} \gamma$ emission line. The position of the radio source B0046-7333 is indicated. The plot extension is $7^{\prime \prime} .07$

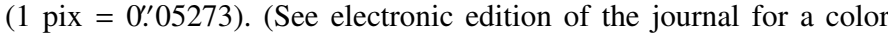
version of this figure.)

Table 3. Relative intensities of IR spectral lines toward N26.

\begin{tabular}{lcccccc}
\hline \hline Component & $\mathrm{He}_{\mathrm{I}}$ & $\mathrm{H}_{2}$ & $\mathrm{H}_{2}$ & $\mathrm{H}_{2}$ & Brr $\frac{\mathrm{HeI} 2.11 \mu \mathrm{m}}{\mathrm{Br} \gamma}$ \\
& $2.11 \mu \mathrm{m}$ & $2.12 \mu \mathrm{m}$ & $2.22 \mu \mathrm{m}$ & $2.24 \mu \mathrm{m}$ & \\
\hline $\mathrm{A}(170)$ & 0.081 & 0.037 & $(1-0) \mathrm{S}(0)(2-1) \mathrm{S}(1)$ & \\
$\mathrm{C}(172)$ & 0.015 & 0.243 & 0.13 & 0.107 & 2.05 & 0.033 \\
\hline
\end{tabular}

observations by Indebetouw et al. (2004). Their work reveals the presence of a compact, radio continuum source (B0046-7333) at RA $0^{\mathrm{h}} 48^{\mathrm{m}} 8.5$, Dec $-73^{\circ} 14^{\prime} 55^{\prime \prime}$, i.e. the position of component $A$. These authors estimated an equivalent spectral type of O7.5 V for the ionizing source of component $\mathrm{A}$.

\subsection{Distribution of $\mathrm{H}_{2}$}

The $\mathrm{H}_{2}$ emission measured toward $\mathrm{N} 26$, in particular component A (Fig. 5), is much weaker than that of $\mathrm{Br} \gamma$ and He I. The $\mathrm{H}_{2}$ profile is composed of two shallow bumps centered around the position of component C. At this position, the profile of $\mathrm{H}_{2}$ decreases slightly. According to Black \& Van Dishoeck (1987), the flux ratios 2-1(S1)/1-0 (S1) and 1-0 (S1)/1-0 (S0) for radiative excitation should range from 0.5 to 0.6 , and from 0.4 to 0.7 , respectively. Our measured ratios are 0.44 for $2-1(\mathrm{~S} 1) / 1-0(\mathrm{~S} 1)$ and 0.53 for $1-0(\mathrm{~S} 1) / 1-0$ (S0) (Fig. 6), in other words not far from the predicted values. This suggests that component $\mathrm{C}$ may be a photodissociation region (PDR). These two weak peaks of $\mathrm{H}_{2}$ emission discovered toward $\mathrm{N} 26$ probably belong to a more extended flat distribution of $\mathrm{H}_{2}$, as detected by Leroy et al. (2007). The presence of such an extended $\mathrm{H}_{2}$ is in line with the result by Israel \& Maloney (2011); in a study of the $\lambda 158 \mu \mathrm{m}$ $\mathrm{C}_{\text {II }}$ line emission, they found PDR parameters for the whole star forming region labeled $\mathrm{N} 22+\mathrm{N} 25+\mathrm{N} 26$, corresponding to almost the whole SMCB2-H II. Rubio et al. (2000) show that $\mathrm{H}_{2}$ knots are associated with clumps of molecular clouds traced by $\mathrm{CO}(2-1)$ and peaks of dust emission, and suggest that massive star formation could be taking place. 


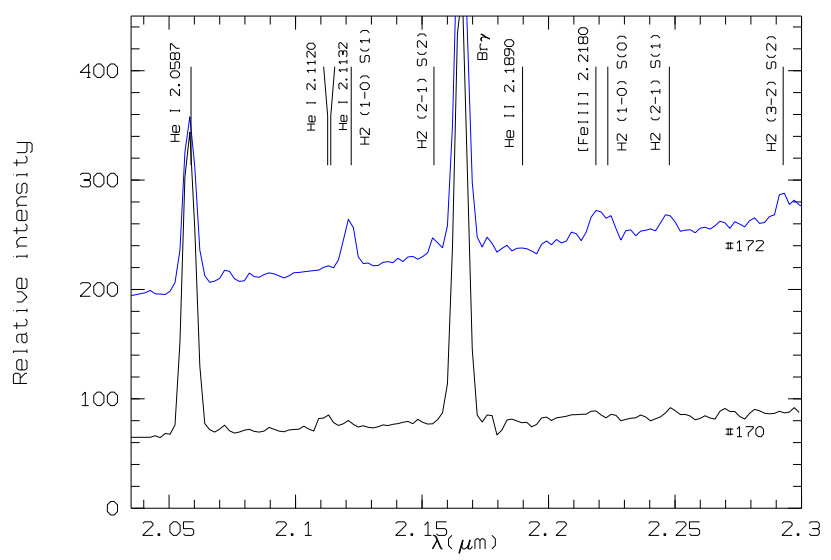

Fig. 6. Spectra of the component A (170) and C (172) extracted from S1.

\subsection{The environment of N26}

\subsubsection{Morphology in the optical and Spitzer images}

The region N26 is located in the northern part of the molecular cloud SMCB2-H II complex, $50 \mathrm{pc} \times 76 \mathrm{pc}$ in size, mapped in the ${ }^{12} \mathrm{CO}(1-0)$ line (Rubio et al. 1993). The H II region lies south of N25, a bright extended circular region $\sim 30^{\prime \prime}$ in diameter (Figs. 1a and 7). The central part of SMCB2-H II is occupied by $\mathrm{N} 22$, the bright, circular and the most extended $\mathrm{H}$ II region of the field, which has a size of $\sim 50$ '. Region N22 is centered roughly on the bright blue star 86 of $V=14.39, B-V=-0.09 \mathrm{mag}$ and fills the cavity between the northern and southern molecular cloud components SMCB2-N and SMCB2-S, respectively. This cavity seems to have been created by hot stars in N22. According to Rubio et al. (1993), the N22 region is comparable to Orion $\mathrm{A}$ as far as the ${ }^{12} \mathrm{CO}(1-0)$ flux is concerned. These authors give for SMCB-N and SMCB2-S a mean radial velocity of $120 \mathrm{~km}^{-1}$ and $126.7 \mathrm{~km}^{-1}$, respectively, slightly smaller than that of N22. We note that N26 is boxed in by the regions N25 and N22. The southern part of SMCB2-H II is composed of N23 and $\mathrm{N} 21$. The $\mathrm{H}_{\mathrm{II}}$ region $\mathrm{N} 23$ has a size of $\sim 25^{\prime \prime}$ and contains the faint blue stars 35,45 , and 54. Region N21, situated west of $\mathrm{N} 23$, is $\sim 15^{\prime \prime}$ in diameter and includes the blue stars 3 and 8 . A faint and compact $\mathrm{H}$ II region of radius 2 '. 6 is detected between $\mathrm{N} 21$ and $\mathrm{N} 23$ in the $\mathrm{H} \beta$ frame (Fig. 7). This region, that we label $\mathrm{N} 23 \mathrm{~A}$, is not detected in the [O III] $\lambda 5007 \AA$ band (Fig. 7). The spectrum presented in Fig. 8a confirms the faint [O III] emission compared to $\mathrm{H} \beta$. This object is probably a low-excitation, compact H II region (Meynadier \& Heydari-Malayeri 2007). Since its spectral lines do not show a significant velocity shift with respect to those of neighboring $\mathrm{H}$ II regions, it should be part of the SMCB2 complex.

\subsubsection{Physical parameters of the $H \|$ regions}

The spectral types of the main ionizing sources of the $\mathrm{H}_{\text {II }}$ regions in the field (Fig. 7) were estimated from UBV photometry (Table 4). We used the main sequence OB star parameters tabulated by Schmidt-Kaler (1982) and the Martins et al. (2005) calibration. We notice that the $\mathrm{O}$ stars classified with Martins et al. are from 0.5 to 2 types hotter. It should be underlined that the classifications, based only on color indices, are uncertain and must be considered as a first attempt. The intrinsic color indices of the $\mathrm{O}$ stars are degenerated; that is, they do not differ much among themselves and are similar to the values of the earliest B
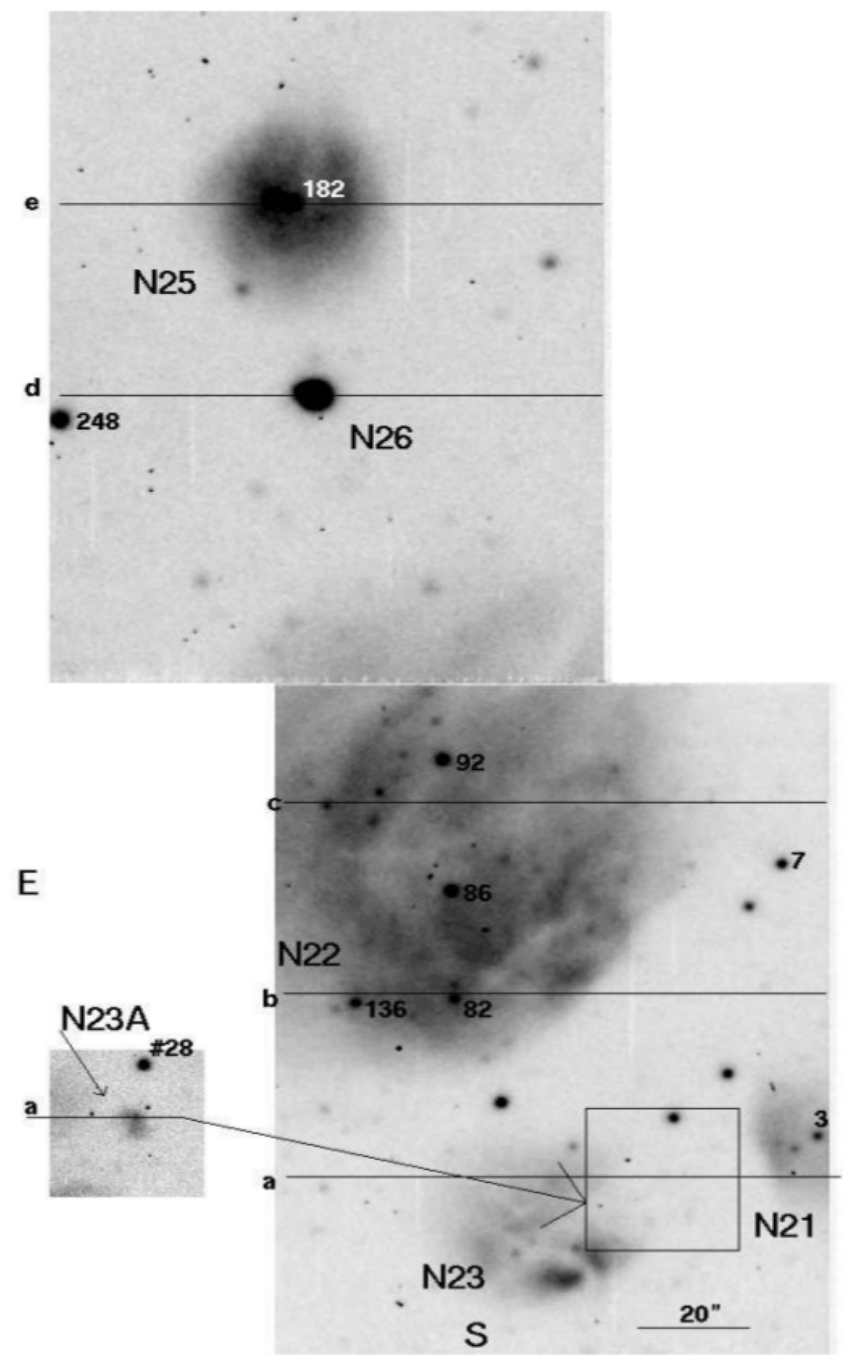

Fig. 7. A mosaic of two [O III] $\lambda 5007$ images showing the SMCB2$\mathrm{H}$ if field. The numbers refer to Table 2. The delineated area in the lower frame does not show nebular emission in [O III] while the same area extracted from the $\mathrm{H} \beta$ image and displayed on the left, shows the new object N23A $\sim 10^{\prime \prime}$ south of star 28 (Fig. 3). The whole [O III] mosaic has a size of the $138^{\prime \prime} \times 264^{\prime \prime}$ or $\sim 42 \mathrm{pc} \times 80 \mathrm{pc}$, while the extracted $\mathrm{H} \beta$ image is $28^{\prime \prime} \times 30^{\prime \prime}$. The $\mathrm{H}$ II regions N21, N22, N23, N25, N26, and the new region $\mathrm{N} 23 \mathrm{~A}$ are indicated. Horizontal lines show the positions $a, b, c, d$, and $e$ of the ESO B\&C long-slit spectroscopic observations. The brighter stars of $V<15.5 \mathrm{mag}$ and $B-V \leq 0 \mathrm{mag}$ are labeled.

main sequence stars (Conti et al. 1986). Moreover, $(B-V)$ and $(U-B)$ values belong to different data sets. The extended $\mathrm{H}$ in region N22 contains 11 blue stars with $B-V \leq 0$, of which the two brightest, 86 and 92, of Type 07 and $05.5 \mathrm{~V}$, respectively, should be the main ionizing sources of N22. The H if region N25 is centered on the bright star 182 whose spectral type is $05.5 \mathrm{~V}$ according to the Martins et al. calibration (Table 4). However, using spectroscopy, this star was classified as O9 V (Hutchings \& Thompson 1988) and O8 V (Testor 2001). In this particular case the spectroscopic result must be preferred. Moreover, the discrepancy between color index and spectroscopic methods point to the uncertainty inherent in using color indices. The main ionization source of $\mathrm{N} 21$ seems to be star 3 of Type O9 or O8.5 V. In N23 the $V$ magnitudes of eight detected stars (Fig. 3) range between $15.93 \mathrm{mag}$ and $18.10 \mathrm{mag}$. Among them stars 31, 35, 


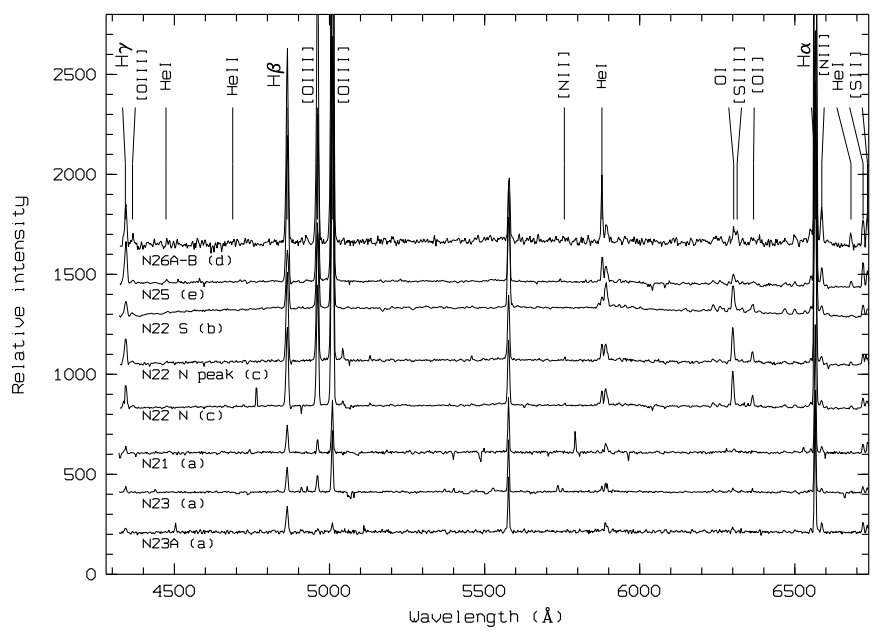

Fig. 8. Optical spectra (sky unsubtracted) crossing the $\mathrm{H}$ in regions N21, $\mathrm{N} 22, \mathrm{~N} 25, \mathrm{~N} 26$, and the faint compact nebula N23A at the positions $a, b, c, d$, and $e$ (Fig. 7).

Table 4. Main ionizing stars of the neighboring $\mathrm{H}_{\text {II }}$ regions.

\begin{tabular}{|c|c|c|c|c|c|c|c|c|}
\hline ID & V & $B-V$ & $U-B$ & $\begin{array}{c}A_{V} \\
(\mathrm{sp})\end{array}$ & $\begin{array}{c}A_{V} \\
\text { (phot) }\end{array}$ & $M_{V}$ & $\begin{array}{c}\text { Spectral } \\
\text { type }^{a}\end{array}$ & $\begin{array}{c}\text { Spectral } \\
\text { type }^{b}\end{array}$ \\
\hline N21 3 & 15.40 & & & 0.69 & & -4.22 & O9 & O8.5 \\
\hline N22 86 & 14.30 & -0.09 & -0.96 & 0.48 & 0.63 & -5 & $\mathrm{O} 7$ & O5.5 \\
\hline N22 92 & 14.25 & -0.19 & -0.96 & 0.57 & 0.27 & -5.25 & O7 & O5 \\
\hline N22 97 & 16.44 & -0.02 & & 0.42 & & -2.91 & B1 & \\
\hline N22 136 & 15.44 & -0.10 & & 0.57 & & -4.06 & BO & O9 \\
\hline N22 155 & 15.98 & -0.19 & & 0.42 & & -3.37 & B0 & \\
\hline N23 35 & 17.60 & -0.24 & & & & & B & \\
\hline N25 182 & 14.25 & -0.13 & -0.95 & 0.33 & 0.48 & -5.01 & O7 & O5.5 \\
\hline
\end{tabular}

Notes. $U-B$ values in Col. 4 are taken from the Massey catalog. In Col. 5, $\mathrm{A}_{V}$ values are derived from the $\mathrm{H} \alpha / \mathrm{H} \beta$ ratios (Table 2). In Col. 6 $\mathrm{A}_{V}$ values are calculated using the $Q$ parameter (Massey et al. 1995). In Col. 7, $M_{V}$ values are estimated using $\mathrm{A}_{V}$ from Col. 5. ${ }^{(a)}$ Spectral types (luminosity class V) derived from Schmidt-Kaler (1982). ${ }^{(b)}$ Spectral types from Martins et al. (2005).

and 59 have a $B-V$ range from $-0.23 \mathrm{mag}$ to $0.01 \mathrm{mag}$, and star 35 may be the main ionization source of the region.

In addition to the stellar content, we investigated the physical properties of the $\mathrm{H}_{\mathrm{II}}$ regions using Boller \& Chivens spectroscopy. From a homogeneous set of CCD frames, onedimensional spectra of N21, N22, N23, N23A, N25, and N26A were extracted by summing a range of pixels along the $4^{\prime \prime}$ eastwest slit. Each range corresponds to the FWHM of the nebula along the slit of the spectra $a, b, c, d$, and $e$ (Fig. 7). Table 5 gives the line ratios from which we derived the physical parameters, such as electron density $\left(N_{\mathrm{e}}\right)$ and temperature $\left(T_{\mathrm{e}}\right)$ of the $\mathrm{H}$ II regions. All the line fluxes were corrected using the coefficient $c(\mathrm{H} \beta)$ derived from the $\mathrm{H} \alpha / \mathrm{H} \beta$ ratio of each spectrum (Fig. 8). We estimate $N_{\mathrm{e}}$ using the five-level atom program by Shaw \& Dufour (1995) from the [S II] doublet $\lambda \lambda 6717 / 6731$ line ratios, assuming $T_{\mathrm{e}}=10^{4} \mathrm{~K}$. We estimate the $T_{\mathrm{e}}$ using the $[\mathrm{O}$ III] $\lambda \lambda 4363 /(4959+5007)$ ratio. Region N22, with an [O III] $(\lambda \lambda 5007+4959) / \mathrm{H} \beta$ ratio of 6 , appears more excited than $\mathrm{N} 21$ and $\mathrm{N} 23$ which have a ratio of about 2.15. The ratio $\mathrm{H} \alpha / \mathrm{H} \beta$ ranges from 3.27 to 4.30 . In Table 5 several other physical parameters are also given.
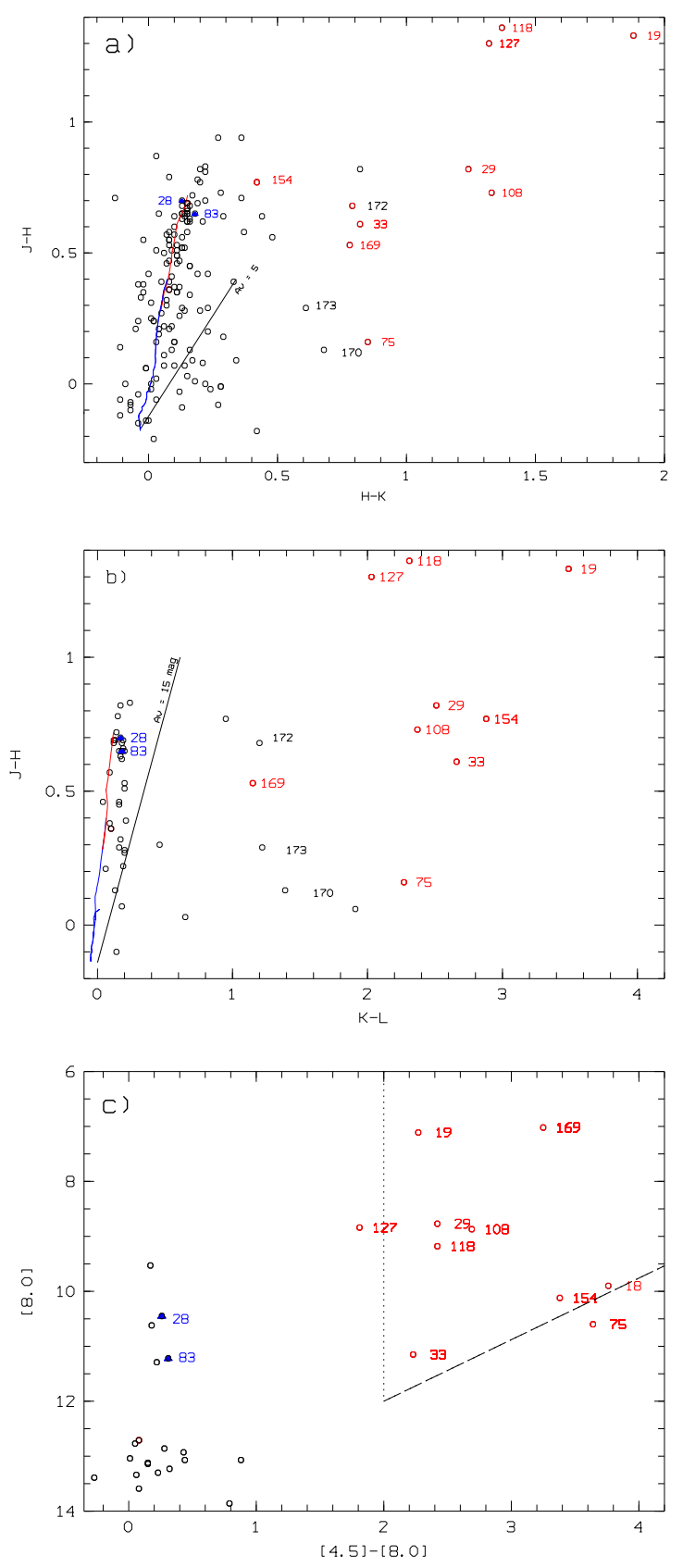

Fig. 9. Infrared photometric diagrams for sources measured toward the SMCB2-H ir complex. See Table 2 for uncertainties. a) $J-H$ versus $H-K$. The blue line is the main sequence isochrone for stars of $1.4 \mathrm{Myr}$ old, and the red line shows the loci of red giants aged 10 Gyr. The black solid line is the reddening vector up to $A_{V}=5 \mathrm{mag}$. The red empty circles correspond to YSO candidates selected using the Whitney et al. (2008) criterion and the blue filled triangles correspond to the Bolatto et al. (2007) criterion (see Sect. 3.5.1). Stars 170, 172, and 173, which are components of the compact $\mathrm{H}_{\text {II }} \mathrm{N} 26$ or star 169 , are indicated, as well as the object 169 , which is the integration of the three mentioned components. b) $J-H$ versus $K-L$. The colors and symbols are the same as those used in panel a). The solid line is the reddening vector up to $A_{V}=15$ mag. c) IRAC [8.0] versus [4.5]-[8.0]. Same colors and symbols as in a). (See electronic edition of the journal for a color version of this figure.)

\subsection{Stellar populations}

The photometric data at our disposal $(B, V, J, H, K ; 3.6,4.5$, $5.8,8.0$, and $24 \mu \mathrm{m}$ ) allow us to identify and study the stellar 
Table 5. H ir region parameters derived from optical emission lines.

\begin{tabular}{|c|c|c|c|c|c|c|c|c|c|c|c|c|c|c|c|}
\hline Spectrum & H II region & $\begin{array}{l}{[\mathrm{O} \text { III] }} \\
\lambda 4363\end{array}$ & $\mathrm{H} \beta$ & $\begin{array}{c}{\left[\mathrm{O}_{\mathrm{IIII}}\right]} \\
\lambda \lambda 4959+5007\end{array}$ & $\mathrm{H} \alpha$ & $\frac{\mathrm{H} \alpha}{\mathrm{H} \beta}$ & $\frac{[\mathrm{OIII}]}{\mathrm{H} \beta}$ & $\frac{[\mathrm{OIII}]}{\lambda 4363}$ & $c(\mathrm{H} \beta)$ & $E(B-V)$ & $\begin{array}{c}\log F(\mathrm{H} \beta) \\
\operatorname{erg~cm}^{-2} \mathrm{~s}^{-1}\end{array}$ & $\begin{array}{c}F W H M \\
\left({ }^{\prime \prime}\right)\end{array}$ & $\frac{\lambda 6717}{\lambda 6731}$ & $\begin{array}{l}T_{\mathrm{e}} \\
(\mathrm{K})\end{array}$ & $\begin{array}{c}N_{\mathrm{e}} \\
\left(\mathrm{cm}^{-3}\right)\end{array}$ \\
\hline (1) & (2) & (3) & (4) & (5) & (6) & (7) & (8) & (9) & (10) & (11) & (12) & (13) & (14) & (15) & (16) \\
\hline $\mathrm{d}$ & N26A-B & 693 & 8019 & 52616 & 44374 & 5.53 & 6.56 & 76 & 0.84 & 0.58 & -12.09 & 2.5 & 0.83 & 12950 & 1216 \\
\hline $\mathrm{e}$ & N25 & 172 & 6597 & 30949 & 21605 & 3.28 & 4.69 & 180 & 0.16 & 0.11 & -12.18 & 30 & 1.31 & 10394 & 110 \\
\hline $\mathrm{c}$ & N22 north* & 177 & 3736 & 22241 & 12492 & 3.34 & 5.95 & 129 & 0.19 & 0.13 & -12.42 & 37 & 1.27 & 11685 & 182 \\
\hline b & $\mathrm{N} 22 \mathrm{~S}$ & 150 & 2841 & 15327 & 10190 & 3.59 & 5.40 & 102 & 0.29 & 0.19 & -12.54 & 50 & 1.31 & 12658 & 110 \\
\hline $\mathrm{a}$ & $\mathrm{N} 23$ & & 909 & 2341 & 3143 & 3.46 & 2.58 & & 0.23 & 0.16 & -13.04 & 30 & 1.25 & & 176 \\
\hline $\mathrm{a}$ & $\mathrm{N} 23 \mathrm{~A}$ & & 1014 & 375 & 3818 & 3.77 & 0.37 & & 0.34 & 0.23 & -12.99 & 8 & 1.34 & & 80 \\
\hline $\mathrm{a}$ & $\mathrm{N} 21$ & & 990 & 2074 & 3705 & 3.74 & 2.10 & & 0.33 & 0.23 & -13.00 & 18 & 0.89 & & 948 \\
\hline
\end{tabular}

Notes. ${ }^{(*)}$ Centered at the position of stars 125 and 155 .

populations lying toward the N26 complex. We also use these data to search for possible YSO candidates. Young stellar objects are enshrouded in dust that absorbs stellar UV and optical radiation and re-radiates in the IR. They can be distinguished from normal stars, i.e., main-sequence, giant, and supergiant stars, by their excess of IR emission. They are positioned in redder parts of the color-color and color-magnitude diagrams. However, the interpretation is not straightforward, because foreground Galactic evolved stars and background external galaxies can display colors similar to those of YSOs, and also because the distribution of circumstellar dust around massive stars is not well known (Stahler et al. 2000; Gruendl \& Chu 2009).

\subsubsection{Photometric diagrams}

On the color-color $J-H$ versus $H-K$ diagram (Fig. 9a) the distribution of the sample stars may be divided into three main groups. The first appears along the main-sequence and redgiant-branch stars with a concentration at $H-K \sim 0.15$ and $J-H \sim 0.65 \mathrm{mag}$. Theoretical models (Lejeune \& Shaerer 2001) indicate the ages of $1.4 \mathrm{Myr}$ and $10 \mathrm{Gyr}$ for the main-sequence stars and red giants, respectively. The $10 \mathrm{Gyr}$ isochrone might indicate an intervening population not physically related to the young massive star region. The second group is a small concentration of faint stars to the right of the reddening vector centered at $H-K \sim 0.2 \mathrm{mag}$. And the third is composed of 13 stars with an IR-excess $H-K>0.6 \mathrm{mag}$. In particular, star 169 includes the sources 170, 172, and 173. Among these 12 red sources, the three reddest, i.e. 19, 118, and 127, have IR excess consistent with those of a circumstellar disk, a protostellar envelope, or a dusty $\mathrm{H}$ II region. The YSO candidates, shown with red empty circles, comply with the following selection criterion: [3.6] $<$ $6.76+1.10 \times([3.6]-[24])$ and [3.6] $<13.86-0.91 \times([3.6]-$ [24]) (Whitney et al. 2008). Candidate carbon stars (the Bolatto et al. 2007 criterion) are also shown and are indicated with filled triangles.

To understand the origin of the dust emission, we include the $L$-band photometry and compare their locations in the $J-H$ versus $K-L$ color-color diagram (Fig. 9b) to known YSO candidates (e.g., Maercker \& Burton 2005), where the blue and red lines represent $3 \mathrm{Myr}$ and $10 \mathrm{Gyr}$ isochrones, respectively (Lejeune \& Shaerer 2001). All 12 red sources have large $(K-L)$ color excess similar to YSO candidates. Four additional sources without excess in $J H K$ colors are found to have $(K-L)$ color excess, 12, 45, 55, and 136; these sources could be AGB or p-AGB stars. To better assess the nature of these red sources, i.e. the origin of dust emission, it is necessary to include midand far-IR photometry.

We now try to confirm these YSO candidates using other diagnostic tools consisting of mid- and far-IR color-magnitude and color-color diagrams. This method has already been employed in several works dealing with YSO candidates (Meixner et al. 2006; Bolatto et al. 2007; Whitney et al. 2008; Gruendl \& Chu 2009; Gordon et al. 2011). Gruendl \& Chu (2009) showed that evolved stars and background galaxies can be excluded from the initial selection of massive YSOs using two simple selection criteria, i.e. [4.5]-[8.0] $\geq 2$ and [8.0] < 14-([4.5]-[8.0]), respectively. Following these criteria, we select eight YSO candidates (Fig. 9c).

All these candidates fall in the loci of class 0 to class II YSOs, following Bolatto et al. (2007). We note that the red object 18 has no $B, V$, and $J H K$ s data and so it is absent in Figs. 9 a and b. For the same reason, it is not listed in Table 6, although the [8.0] versus [4.5]-[0.8] plot suggests it as a possible YSO candidate (Fig. 9c). The enclosed area shows the sample that does not include normal and asymptotic giant branch stars, nor background galaxies. The eight YSO candidates are found in the upper-right wedge. The red dots are the YSO candidates selected using the above-mentioned criterion by Whitney et al. (2008).

It should also be mentioned that we had to calculate the IRAC photometry of star 18 , because in the SAGE catalog only $4.5 \mu \mathrm{m}$ and $5.8 \mu \mathrm{m}$ values were available. Therefore, we obtained the photometry of the $3.6 \mu \mathrm{m}$ to $8.0 \mu \mathrm{m}$ bands using profiles instead of apertures, because 18 is a blended source, as indicated by its FWHM which is much larger than the IRAC PSF. The profile method yields more accurate sky subtraction in the wings. The method consisted of crossing several cuts through 18 along rows and integrating the fluxes in each band. In order to calibrate the photometry, the operation was also carried out for star 67 (Table 2). The uncertainties in the $3.6 \mu \mathrm{m}$ to $8 \mu \mathrm{m}$ bands are $\leq 0.05 \mathrm{mag}$. We note that the $24 \mu \mathrm{m}$ measurement of source 18 might include the nearby source 14 as these IRAC sources are unresolved by the MIPS $24 \mu \mathrm{m}$ PSF.

The red stars 75 and 127 in Fig. 9c are slightly outside the limits of the YSO area. However, in the [3.6]-[8.0] versus [8.0]-[24] color-color diagram (not shown), they are inside the area and may therefore be classified as YSO candidates. Moreover, objects 170, 172, and 173 are absent in Fig. 9c because they lack mid-IR data. Figure 9c also shows that most of the stars are located along a vertical band with [4.5]-[8.] ranging from $\sim 0$ to 0.6. According to Gordon et al. (2011), most of these stars occupy the loci of $\mathrm{O}$ and early B stars. 
Table 6. Parameters derived from SED fits to the YSO candidates.

\begin{tabular}{|c|c|c|c|c|c|c|c|c|c|c|c|}
\hline ID & $\alpha$ & $\begin{array}{c}M_{*} \\
\left(M_{\odot}\right)\end{array}$ & $\begin{array}{l}\Delta M_{*} \\
\left(M_{\odot}\right)\end{array}$ & $\begin{array}{l}L_{\text {tot }} \\
\left(L_{\odot}\right)\end{array}$ & $\begin{array}{l}\Delta L_{\mathrm{tot}} \\
\left(L_{\odot}\right)\end{array}$ & $\begin{array}{l}M_{\text {disk }} \\
\left(M_{\odot}\right)\end{array}$ & $\begin{array}{c}\Delta M_{\text {disk }} \\
\left(M_{\odot}\right)\end{array}$ & Stage & $\Delta$ Stage & Type & Remarks \\
\hline (1) & $(2)$ & (3) & (4) & (5) & (6) & (7) & (8) & (9) & (10) & (11) & (12) \\
\hline 19 & $004752.37-731712.85$ & 17.4 & 3.0 & 22000 & 8400. & $5.2 \mathrm{E}-02$ & $1.4 \mathrm{E}-01$ & 1.0 & 0.0 & I & between N21 and N23 \\
\hline 29 & $004753.61-731710.22$ & 14.0 & 2.7 & 9300 & 3700. & $8.1 \mathrm{E}-02$ & $1.7 \mathrm{E}-01$ & 1.1 & 0 . & I & . \\
\hline 33 & $004754.96-731724.72$ & 9.6 & 1.0 & 3100 & 1100. & $5.3 \mathrm{E}-02$ & $1.1 \mathrm{E}-01$ & 1.1 & 0.2 & I & $"$ \\
\hline 75 & $004801.27-731659.22$ & 7.1 & 1.1 & 1300 & 350. & $6.0 \mathrm{E}-02$ & 4.4E-02 & 1.0 & 0.0 & II & between N22 and N23 \\
\hline 108 & $004804.39-731521.60$ & 12.7 & 1.3 & 13000 & 4400. & $1.3 \mathrm{E}-01$ & 2.6E-01 & 1.4 & 0.5 & I & $\mathrm{N}$ border of $\mathrm{N} 22$ \\
\hline 118 & $004804.90-731755.92$ & 15.4 & 3.4 & 19000 & 17000. & $1.1 \mathrm{E}-01$ & 2.1E-01 & 1.0 & 0.2 & I & SE border of N23 \\
\hline 127 & $004805.68-731744.36$ & 9.9 & 1.7 & 5800 & 2100 & 8.9E-02 & $1.3 \mathrm{E}-01$ & 1.5 & 0.5 & II & \\
\hline 154 & $004807.61-731428.19$ & 10.5 & 1.0 & 7300 & 2100. & $9.3 \mathrm{E}-03$ & 7.7E-03 & 2.0 & 0.0 & II & SW border of N25 \\
\hline 169 & $004808.60-731454.69$ & 24.8 & 1.4 & 80000 & 12000. & $1.6 \mathrm{E}-01$ & $2.8 \mathrm{E}-01$ & 1.5 & 0.5 & I & $\mathrm{N} 26, \mathrm{H}_{\text {II }}$ region, multiple \\
\hline
\end{tabular}

\subsubsection{Massive YSO candidates}

We have selected 12 possible YSO candidates after applying several diagnostics tools, but only 9 of them, those with mid-IR detection, will be investigated further with spectral energy distribution (SED) fitting. Most of these objects should not be low-mass protostars, but rather massive YSOs. The absolute magnitudes in the $L$ band for our candidates range from -8.4 to -4.5 . In comparison, the $L$ absolute magnitudes for 58 low-mass YSO candidates in the Orion Trapezium cluster are found to be between -3.5 and 4.2. These comparatively large magnitudes and the red colors that characterize massive YSOs are also notable in the color-magnitude diagrams, for example the [8.0] versus [4.5]-[8.0] (Fig. 9c). It should be pointed out that the YSO SED fitting, and consequently the parameters obtained, are formal results, i.e. mere suggestions from the physical point of view.

Low-mass YSOs are divided into classes based on their observed spectral indices (Lada 1987). In contrast, we do not yet have a well-defined classification system for massive YSOs. Therefore, we use the commonly accepted scheme of low-mass YSOs as a starting point to look into the physical parameters of high mass YSOs. The geometry of dust disk and envelope applicable to low-mass YSOs has been adopted in radiative transfer models of high-mass YSOs (e.g., Whitney et al. 2004; Robitaille et al. 2006).

Robitaille et al. (2006) suggested a physical classification scheme for YSOs of all masses. This classification is analogous to the Class scheme for low-mass YSOs, but uses physical quantities, instead of the slope of the SED to define the evolutionary stage of the models. This classification scheme, while physical, is model dependent, and the comparison with observations requires data taken with sufficient angular resolutions to distinguish them among individual sources and from backgrounds, which is not always the case for MC objects. Chen et al. (2009) suggested an empirical classification of the observed SEDs of massive YSOs, and used a "Type" nomenclature to distinguish from the "Classes" for low-mass YSOs and the "Stages" for model YSOs. According to this empirical classification, massive YSOs are divided into Types I-III.

Type I YSOs have SEDs with a steep rise from the nearIR to $24 \mu \mathrm{m}$, which is caused by radiation from their circumstellar envelopes. They are visible in the $K$ band and bright at $24-70 \mu \mathrm{m}$, but are generally not visible in the optical or $J$ band. Type I YSOs are often found in or behind dark clouds.

Type II YSOs have SEDs with a low peak in the optical and brighten up from $J$ to $8 \mu \mathrm{m}$. Type II has a high peak at $8-24 \mu \mathrm{m}$ and then faint again at $24 \mu \mathrm{m}$.
Type III YSOs have bright SEDs in the optical, with a modest emission of dust in the near- to mid-IR, corresponding to remnant circumstellar material. Their brightness fades in the longer wavelengths, and they are often surrounded by $\mathrm{H}$ in regions.

Following the same procedure detailed in Chen et al. (2009) to infer the physical parameters for the YSOs, we compare the observed SEDs to model SEDs and select best-fit models based on minimum $\chi_{\min }^{2}$. We use a large model grid containing 20000 pre-calculated dust radiative transfer models and a fitting code from Robitaille et al. (2007). The fitting requires input parameters including the fluxes of a YSO and uncertainties of the fluxes. The uncertainties of the fluxes are specified in Sects. 2.1.1 and 2.1.2 and are listed in Table 2.

Figure 10 shows the best-fitting model (plotted in black lines) for each source, but typically there is a range of models that are nearly as consistent with the data, i.e. with $\chi^{2}$ not significantly greater. We have used a cutoff of $\chi^{2}-\chi_{\min }^{2} \leq 3$ per data point for these acceptable models, and plot them in gray. The results of the model fits are given in Table 6 which lists selected physical parameters: weighted averages and standard deviations of central stellar mass $\left(M_{\star}\right)$, total luminosity $\left(L_{\text {tot }}\right)$, and disk mass $\left(M_{\text {disk }}\right)$. These averages and standard deviations, shown with the $\Delta$ sign, are calculated from best-fit and acceptable models using the inverse square of $\chi^{2}$ as the weight; they show a possible range of the physical parameters of a YSO. For each accepted model, the evolutionary stage is determined using $\dot{M}_{\text {env }} / M_{\star}$ and $M_{\text {disk }} / M_{\star}$ ratios as defined in Robitaille et al. (2006) and the range of the evolutionary stage, known as the stage range, is determined from the standard deviation of all stages inferred for a YSO.

It turns out that seven candidates have a mass $\geq 10 M_{\odot}$ (Table 6). The most remarkable massive YSO candidates in the sample are 19,29, and 118 with estimated masses of $\sim 17,14$, and $15 M_{\odot}$, respectively, and are all of Type I. These are very bright objects in the IRAC bands. They are situated in the southern concentration of the molecular cloud shell. Sources 19 and 29 are adjacent objects lying near the newly detected $\mathrm{H}$ II region N23A (Figs. 3 and 1). Object 118 is situated east of N23. Table 6 also includes object 169 which is the integration of the three sources 170, 172, and 173 comprising the compact $\mathrm{H}$ II region N26. We will address this object in the next section.

\section{Discussion}

The main object of this study, N26, is situated in the northern part of the molecular cloud surrounding the central cavity. It probably represents the youngest massive star formation accessible to optical and infrared wavelengths in this region. It 

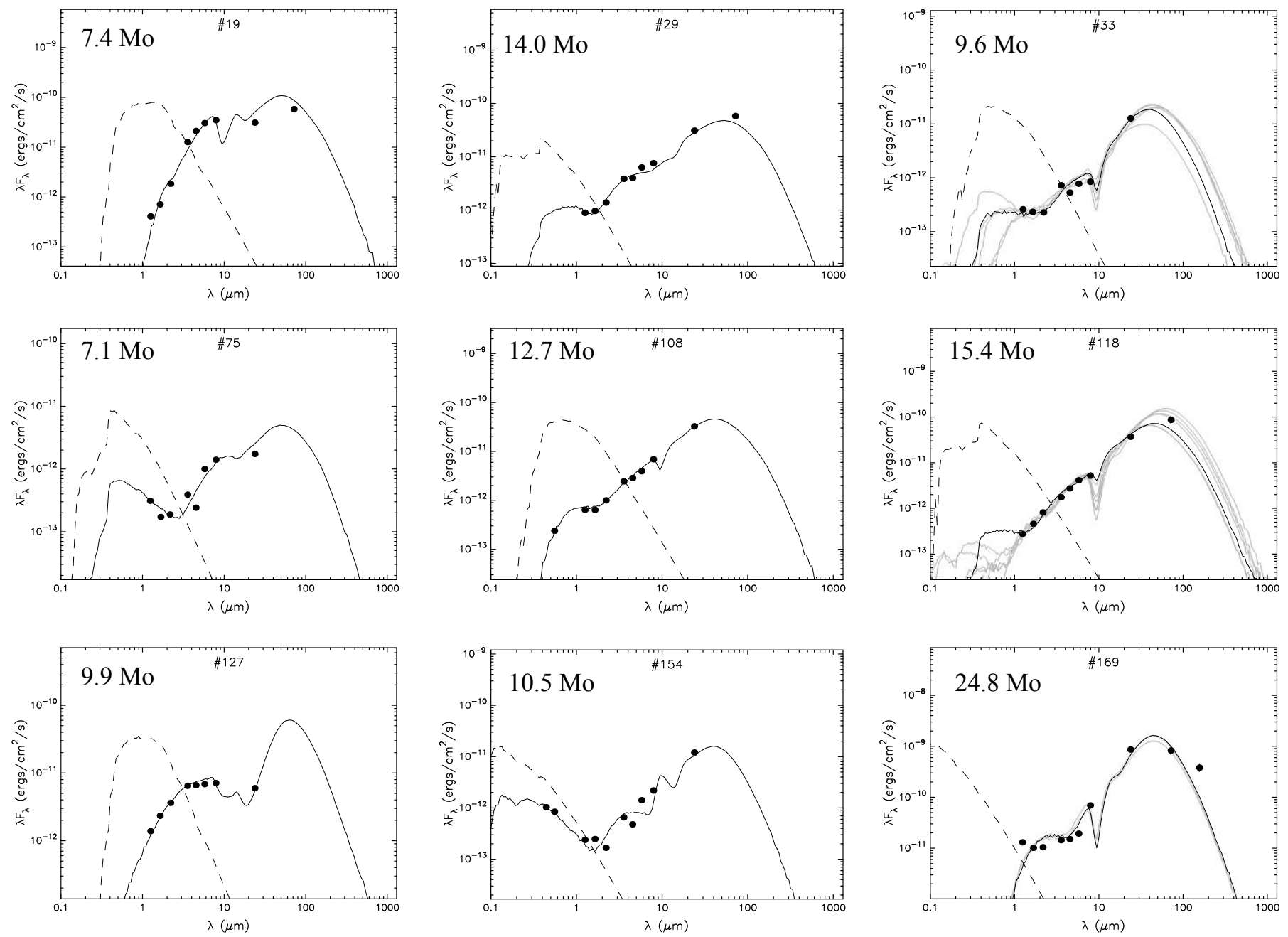

Fig. 10. Spectral density distribution of nine YSOs obtained using the YSO models of Robitaille et al. (2006). Filled circles are the flux values converted from magnitudes listed in Table 2 . The thin black line represents the best fitting SED, and the gray lines show all models that also fit the data well (defined by $\chi^{2}-\chi_{\text {best }}^{2}$, where $\chi^{2}$ is the value per data point). The dashed line represents the stellar photosphere model (corrected for the foreground extinction). The derived masses are indicated; see Table 6 for other parameters.

belongs to a small class of compact, HEBs in the MCs of which only five other examples are presently known in the SMC: N88A (Testor et al. 2010), N81 (Heydari-Malayeri et al. 1999), N66A (Heydari-Malayeri \& Selier 2010), N33 (Selier et al. 2011), and N77A (Selier \& Heydari-Malayeri 2012). This region has the particularity of being one of the most compact members of this class. Some observational data presented in this paper suggest that the $\mathrm{H}$ II region is powered by a massive star of spectral type 07-09 V.

In comparison with Galactic regions, some of the HEBs are similar to classical $\mathrm{H}_{\text {II }}$ regions and some look like compact $\mathrm{H}$ II regions detected in the radio continuum (Martin-Hernandez et al. 2005). However, HEBs and their low-excitation counterparts, called LEBs (Meynadier \& Heydari-Malayeri 2007), should be considered in the context of massive star formation in the MCs. It would be interesting to know in what physical conditions these younger and less populous massive star forming regions are born adjacent to typical giant $\mathrm{H}_{\text {II }}$ regions in the MCs. Given the difficulty in observing the MC HEBs and LEBs, hence the small known sample, it would be helpful to study similar objects in our Galaxy for which higher resolution observations are possible. To our knowledge, no research has been undertaken yet to find and study compact $\mathrm{H}_{\text {II }}$ regions in the vicinity of Galactic giant $\mathrm{H}_{\text {II }}$ regions, such as NGC 3603, M8, M17, and others (Damineli et al. 2005). As a first step, it seems helpful to identify Galactic high-excitation $\mathrm{H}$ II regions in the peripheral zone of giant $\mathrm{H}$ II regions and look into their physical characteristics. A study of this kind will constitute a research project on its own, to which enough time and effort should be devoted. This study may, however, face two notable handicaps: distance uncertainties involved in Galactic H II region studies and the difficulty of measuring the total luminosity of Galactic objects extending over several arcminutes on the sky. We note that Galactic H II regions like Orion, Sh2156, Sh2-152, Sh2-269, and RCW34 (Heydari-Malayeri 1988; Heydari-Malayeri \& Testor 1981; Simpson 1973; HeydariMalayeri et al. 1980, 1982) have the same physical size and excitation as the MC LEBs. It remains to identify their higher excitation counterparts in the adjacent areas of giant $\mathrm{H}$ II regions.

The region N26 may be associated with three YSOs, 170 (component A), 173 (component B), and 172 (component C); the third is a massive one, as suggested by SED fitting of the available data (not shown in Fig. 10). However, the problem is that the Spitzer IRAC bands do not have the necessary spatial resolution and moreover the SEDs lack data in the near- and midIR spectral ranges. The absence of the [4.5], [5.8], [8], [24], and 
[70] $\mu \mathrm{m}$ for components $\mathrm{A}, \mathrm{B}$, and $\mathrm{C}$, and also $B V$ bands for $\mathrm{C}$ (Table 2), makes the construction of the SED incomplete and hence difficult to compare with models.

Since we lack mid-IR Spitzer data for the SED fitting of each of the N26 components A, B, and C, we tried to perform the SED fitting on the whole integrated object 169 , which contains the three components 170, 172, and 173. The whole of object 169 measures $\sim 4^{\prime \prime} \times 2{ }^{\prime \prime} .5$, corresponding to $1.2 \times 0.8 \mathrm{pc}$. The mass of the associated YSO turns out to be $24.8 \pm 1.4 M_{\odot}$ (Table 6 ), which is much higher than the individual masses of the three YSO candidates in components A, B, and C. The model fitting to the SED of combined fluxes from several YSOs would result in a higher inferred stellar mass than that to each of these individual YSOs, as demonstrated in Chen et al. (2010). In addition, extra uncertainties could incur in fitting to these individual YSOs because of their lack of $24 \mu \mathrm{m}$ measurements, as the very young ones have SEDs peaking at $24 \mu \mathrm{m}$ or even longer wavelengths (Chen et al. 2009, 2010). Most interestingly, the inferred mass of $24.8 \pm 1.4 M_{\odot}$ from the integrated SED is consistent with that of the $07-9 \mathrm{~V}$ ionizing star determined from our Near-IR spectra. It appears that the mid-IR luminosity is dominated by this compact HEB ionized by the most massive star $(\mathrm{O} 7-9 \mathrm{~V})$ in this group of stars.

It is not surprising, therefore, that N26 hosts a massive YSO in its component $\mathrm{C}$, because N26 is a very young massive star formation region. In similar cases, YSOs have already been reported to be associated with other HEBs, for example the LMC objects N159-5 (Chen et al. 2010) and N11A (Vaidya et al. 2009 ), in spite of a spatial resolution of $\geq 0.5 \mathrm{pc}$. Moreover, thanks to a high spatial resolution of $\sim 0.06 \mathrm{pc}$, YSO candidates have been located in the SMC HEB N88A (Testor et al. 2010). Hence, the presence of this YSO is compatible with the fact that HEBs are sites of ongoing massive star formation.

This area of the SMC is a very interesting site of massive star formation with respect to a broader view of the environment in which N26 lies. This statement is supported by the presence of several $\mathrm{H}$ II regions and numerous OB stars associated with a giant molecular cloud, as presented in the preceding sections. Massive star formation has probably developed from the main $\mathrm{H}_{\text {II }}$ region N22, which, with its several OB stars, has disrupted the molecular cloud and has created a central cavity in the SMCB2-H II complex. This suggests that the formation of massive stars has probably propagated from N22 in both north and south directions.

This picture is consonant with the detection of the eight YSOs candidates toward the SMCB2-H II complex, among which are seven massive candidates. The most massive candidate in the sample, object 19 , has a mass of $17.4 \pm 3.0 M_{\odot}$ (Table 6) and lies in the direction of the southern molecular ridge, between the $\mathrm{H}_{\text {II }}$ regions N21 and N23. This object is situated at about 4.' 5 from object 29 , which is the second most massive candidate in the sample, with a mass of $14.0 \pm 2.7 M_{\odot}$. There are four other YSO candidates in that direction: 33, 75, 118, and 127 . The presence of these massive YSOs supports the observation that the southern concentration of the SMCB2 molecular cloud bordering the central N22 cavity is an active site of massive star formation.

The other YSO candidates belong to the northern concentration of the molecular cloud, where the compact H II region N26 is located. The most massive object in this group, i.e. 108, lies about $30^{\prime \prime}$ (9 pc) south of N26 and has a mass of $12.7 \pm 1.3 M_{\odot}$ (Table 6). Interestingly, this object lies at the northern border of the central cavity. Although geometrical coincidence cannot be firmly excluded, and we cannot reject the possibility that N22 simply erodes the less dense part of the clump that forms YSOs, there is a probability that the formation of this object is related to northward expansion of the $\mathrm{H}_{\mathrm{II}}$ region $\mathrm{N} 22$. The absence of YSOs in the direction of N22 is due to the fact that there is no notable concentration of molecular gas and dust there. Indeed massive stars of N22 have disrupted the molecular cloud, creating the cavity visible on the Spitzer images (Fig. 1).

More generally, apart from the lack of data, some words of caution are in order as to the method used in describing the characteristics of the YSOs. The point spread function (PSF) of the IRAC bands at the SMC distance of $61 \mathrm{kpc}$ is $\sim 2^{\prime \prime}$, corresponding to $\sim 0$.' $6 \mathrm{pc}$. The angular resolution for the MIPS bands is even lower, ranging from $\sim 1.8 \mathrm{pc}$ to $12 \mathrm{pc}$ for the $24 \mu \mathrm{m}$ and $160 \mu \mathrm{m}$ bands, respectively. Even with a resolution of $0.6 \mathrm{pc}$, which is the size of a whole cluster, we may be probing the combined effects of the embedded components. For comparison, the Orion core, which includes many various components, among which the Trapezium cluster and BN/IRc2 (YSO), has a size of only $\sim 0.5 \mathrm{pc}$. Moreover, since we are dealing with $\mathrm{H}$ II regions, an $\mathrm{H}$ II region taken globally should contain several components such as strong nebular emission, UV photons acting on dust concentrations (accompanied by secondary IR emission), radio emission, embedded clusters, etc. More specifically, the contribution from polycyclic aromatic hydrocarbon emission to the emission at IRAC wavelengths is significant, particularly in the $8 \mu \mathrm{m}$ band, making the sources easy to confuse with YSOs. Therefore, we must be very cautious in interpreting the present data.

With the above precautions in mind, we now underline another point in favor of the presence of the YSO candidates. The SMCB2-H II complex is a star forming region enclosing strong ionized hydrogen emission, dust concentrations, and hundreds of stars. We have detected only eleven YSOs toward this complex. The massive candidates are also associated with the most recent massive star formation events detectable in the optical and infrared bands. It appears that massive stars tend to form where massive star formation has occurred before, a trend seen in other LMC H II regions (Chen et al. 2009, 2010). These sites already had the conditions to form massive stars in the past and hence unsurprisingly continue to form new massive YSOs.

\section{Concluding remarks}

The region N26 is a member of the rare category of highexcitation blobs in the SMC. We have presented high spatial resolution imaging of this object in the $J H K \mathrm{~s} L$ '-bands with a FWHM of $\sim 0$ !' $1-0$ ' 35 . Imaging with a lower spatial resolution is also presented in $B, V$, near-IR, mid-IR, $24 \mu \mathrm{m}$, and $70 \mu \mathrm{m}$ for both N26 and its surrounding regions, which are associated with the molecular cloud SMCB2. Component A, which is $\sim 1^{\prime \prime}$, in size, contains at least two stars, one of which, A1, with a spectral type of O7-O9, might be the main exciting source of N26. Moreover, component A coincides with the radio continuum source B0046-7333. On the other hand, a bright component, C, which lies between A and B and has no optical counterpart, is uncovered for the first time. In general, N26 appears to contain at least five stars superimposed on a very small cluster of faint stars that are not very well resolved between component $\mathrm{A}$ and star 160 in a $\sim 4^{\prime \prime}$, area. Components $\mathrm{A}$ and $\mathrm{C}$ are also found to be associated with several stars each. Massive star formation is probably still going on in N26, if the detection of the massive YSO (172) is confirmed. This object is the most massive member of the massive YSOs candidates in the SMCB2. 
Mid-IR images of the molecular cloud SMCB2 on a larger scale of $50 \mathrm{pc} \times 76 \mathrm{pc}$ display a shell structure of gas and dust surrounding a central cavity created by $\mathrm{N} 22$, the main $\mathrm{H}$ in region of the molecular complex. The region N26, which is associated with the northern concentration of the shell, is the most compact and excited HiI region of the complex. To check the presence of massive YSOs in other parts of the molecular cloud, we used selection criteria based on color-magnitude and color-color diagrams in various bands $(B, V, L, J, H, K \mathrm{~s}, 3.6,4.5,8.0,24$, 70 , and $160 \mu \mathrm{m}$ ), and then SED fitting on the qualified objects. All the YSO candidates are associated with the two northern and southern concentrations or shells of the molecular complex SMCB2 bordering the central cavity. The southern concentration, notably N23, harbors more massive YSO candidates than the northern concentration. A faint and compact $\mathrm{H}$ II region with weak [O III] $\lambda 5007$ emission is also detected toward this part of the complex.

We are aware that the low spatial resolution at the distance of the $\mathrm{SMC}$, in addition to contamination by dust and gas emission, makes the detection and classification of YSOs questionable. However, taking the necessary precautions, we have to push the exploitation of the data to their limit in order to study massive star formation in metal deficient galaxies, the nearest example of which is the SMC.

Acknowledgements. We would like to thank an anonymous referee whose comments contributed to improve the presentation. M.S. acknowledges financial support from the NASA ADAP award NNX11AG50G. This research has made use of the SIMBAD data base, operated at CDS, Strasbourg, France.

\section{References}

Allen, L. E., Calvet, N., D'Alessio, P., et al. 2004, ApJS, 154, 363

Bica, E. D., \& Dutra, C. M. 2000, AJ, 119, 1214

Black, J. H., \& van Dishoeck, E. F. 1987, ApJ, 322, 412

Bolatto, A. D., Simon, J. D., Stanimirovic, S., et al. 2007, ApJ, 655, 212

Bot, C., Rubio, M., Boulanger, F., et al. 2010, A\&A, 524, A52

Bonanos, A.Z, Lennon, D. J., Kohlinger, F., et al. 2010, AJ, 140, 416

Chen, C. H. R., Chu, Y.-H., Gruendl, R. A., Gordon, K. D., \& Heisch, F. 2009, ApJ, 695, 511

Chen, C. H. R., Indebetouw, R., Chu, Y.-H., et al. 2010, ApJ, 721, 1206

Conti, P. S., Garmany, C. D., \& Massey, P. 1986, AJ, 92, 48

Cutri, R. M., Skrutskie, M. F., van Dyk, S., et al. 2003, The IRSA 2MASS All-Sky Point Source Catalog, NASA/IPAC Infrared Science Archive Vizier On-line Data Catalog: I: 246, http://irsa.ipac.caltech.edu/ applications/Gator/

Damineli, A., Blum, R. D., Figueredo, E., \& Conti, P. S. 2005, in Massive Star Birth: A Crossroads of Astrophysics, eds. R. Cesaroni, M. Felli, E. Churchwell, \& M. Walmsley (Cambridge: Cambridge University Press), IAU Symp. Proc., 227, 407

Davies, R. D., Elliot, K. H., \& Meaburn, J. 1976, MNRAS, 81, 89

Dufour, R. J. 1984, in Structure and Evolution of the Magellanic Clouds, IAU Symp. 108, 353

Gordon, K. D., Meixner, M., Meade, M. R., et al. 2011, AJ, 142, 102

Gruendl, R. A., \& Chu, Y. 2009, ApJS, 184, 172

Hanson, M., Luhman, K., \& Rieke. 2002, ApJS, 138, 35

Henize, K. G. 1956, ApJS, 2, 315

Heydari-Malayeri, M. 1988, A\&A, 202, 240
Heydari-Malayeri, M., \& Selier, R. 2010, A\&A, 517, A39

Heydari-Malayeri, M., \& Testor, G. 1981, A\&A, 96, 219

Heydari-Malayeri, M., \& Testor, G. 1982, A\&A, 111, L11

Heydari-Malayeri, M., Testor, G., \& Lortet, M. C. 1980, A\&A, 84, 154

Heydari-Malayeri, M., Testor, G., Baudry, A., et al. 1982, A\&A, 113, 118

Heydari-Malayeri, M., Jarvis, B., \& Gilliotte, A. 1989, in The Boller \& Chivens Spectrographs (ESO Operating Manual), 9

Heydari-Malayeri, M., Rosa, M. R., Charmandaris, V., Deharveng, L., \& Zinneker, H. 1999, A\&A, 352, 665

Heydari-Malayeri, M., Rosa, M. R., Charmandaris, V., et al. 2010, in The Impact of HST on European Astronomy, ed. F. D. Macchetto, Astrophys. Space Sci. Proc., 31

Hutchings, J. B., \& Thompson, I. B. 1988, ApJ, 331, 294

Indebetouw, R., Johnson, K., \& Conti, P. 2004, AJ, 128, 2206

Israel, F. P. 1997, A\&A, 328, 471

Israel, F. P., \& Maloney, P. R. 2011, A\&A, 531, A19

Kato, D., Nagashima, C., Nagayama, T., et al. 2007, PASJ, 59, 615

Keller, S. C., \& Wood, P. R. 2006, ApJ, 642, 834

Lada, C. J. 1987, in Star Forming Regions, eds. M. Peimbert, \& J. Jugaku (Dordrecht: D. Reidel Publishing Co), IAU Symp., 115, 1

Lada, C. J., A. A Muench, Haisch, Karl, E., Jr, et al. 2000, ApJ, 120, 3162

Lawton, B., Gordon, K. D., Babler, B., et al. 2010, ApJ, 716, 453

Lejeune, T., \& Shaerer, D. 2001, A\&A, 366, 538

Leroy, A., Bolatto, A., Stanimirovic, S., et al. 2007, ApJ, 658, 1027

Maercker, M., \& Burton, G. 2005, A\&A, 438, 663

Martin-Hernandez, N. L., Vermeij, R., \& van der Hulst, J. M. 2005, A\&A, 433, 205

Martins, F, F., Shaerer, D., \& Hillier, D. J. 2005, A\&A, 436, 1049

Massey, P. 2002, ApJS, 141, 81

Massey, P., Lang, C., Degi-Eastwood, K., \& Garmany, C. 1995, ApJ, 438, 188

Meixner, M., Gordon, K. D., Indebetouw, R., et al. 2006, AJ, 132, 2268

Meynadier, F., \& Heydari-Malayeri, M. 2007, A\&A 461, 565

Mizuno, N., Rubio, M., Mizuno, A., et al. 2001, PASJ, 53, L45

Peña-Guerrero, M. A., Peimbert, A., Peimbert, M., \& Ruiz, M. T. 2012, ApJ, 746,115

Rieke, G., Young, E. T.,Engelbracht, C. W., et al. 2004, ApJS, 154, 25

Robitaille, T. P., Whitney, B. A., Indebetouw, R., Wood, K., \& Denzmore, P. 2006, ApJS, 167, 256

Robitaille, T. P., Whitney, B. A., Indebetouw, R., \& Wood, K. 2007, ApJS, 169 328

Rubio, M., Lequeux, J., Boulanger, F., et al. 1993, A\&A, 271, 1

Rubio, M., Contursi, A., Lequeux, J., et al. 2000, A\&A, 359, 1139

Schaefer, B. E. 2008, AJ, 135, 112

Selier, R., \& Heydari-Malayeri, M. 2012, A\&A, 545, A29

Selier, R., Heydari-Malayeri, M., \& Gouliermis, D. A. 2011, A\&A, 529, A40

Shaw, R. A., \& Dufour, R. J. 1995, PASP, 107, 896

Schmidt-Kaler, T. 1982, Landolt-Bornstein, New Series, Group VI, 2, 14

Simpson, J. P. 1973, PASP, 85, 479

Stahler, S., W., Palla, F., \& Ho, P. T. P. 2000, in Protostars and Planets IV, eds. V. Mannings, A. Boss, \& S. Russell (Tucson: Univ. Arizona Press), 327

Stetson, P. B. 1987, PASP, 99, 191

Testor, G. 2001, A\&A, 372, 667

Testor, G., Lemaire, J. L., Heydari-Malayeri, M., et al. 2010, A\&A, 510, A95

Vaidya, K., Chu, You-Hua, Gruendl, R. A., Chen, C.-H. R., \& Looney, L. W. 2009, ApJ, 707, 1417

Westerlund, B. E. 1997, in The Magellanic Clouds (Cambridge: Cambridge Univ. Press), 28

Whitney, B. A., Indebetouw, R., Bjorkman, J. E., \& Wood, K. 2004, ApJ, 617, 1177

Whitney, B., Sewilo, M., Indebetouw, R., et al. 2008, AJ, 681, 1341

Wilke, K., Stickel, M., Hass, M., et al. 2003, A\&A, 401, 873 